\title{
Synthesis of Bicyclic $\gamma$-Ylidenetetronates
}

\author{
Francisco Velázquez and Horacio F. Olivo* \\ Division of Medicinal and Natural Products Chemistry, College of Pharmacy \\ The University of Iowa, Iowa City, IA 52242
}

\section{Supplementary Material}

General Procedures. ${ }^{1} \mathrm{H}$ and ${ }^{13} \mathrm{C}$ NMR spectra were obtained using Brucker WM-360 and AMX-400 spectrometers. Carbon multiplicities were determined using DEPT experiments. IR spectra were recorded using a Nicolet 210 spectrometer. Melting points were obtained in a Thomas Hoover Melting Point Apparatus. Melting points reported here are uncorrected. Analytical TLC was performed using pre-coated silica gel $60 \mathrm{~F}_{254}$ Merck plates.

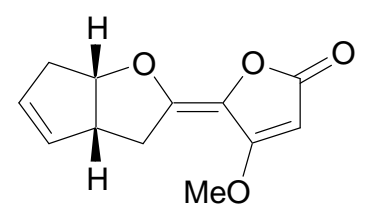

$\boldsymbol{Z}$ - $\boldsymbol{\gamma}$-Ylidenetetronate $\boldsymbol{Z}$-24: $\boldsymbol{R}_{\mathbf{f}}=0.25$ (ethyl acetate/petroleum ether (1:1); UV detection); m.p. $=140-141{ }^{\circ} \mathrm{C}$, IR (KBr) 3119, 2950, 1733, 1693, 1585, 1195, 1069, 844 $\mathrm{cm}^{-1} ;{ }^{1} \mathbf{H}-\mathbf{N M R}\left(400 \mathrm{MHz}, \mathrm{CDCl}_{3}\right) \delta 5.75(1 \mathrm{H}, \mathrm{ddd}, J=2.2,2.3,7.4 \mathrm{~Hz}), 5.55(1 \mathrm{H}, \mathrm{ddd}$, $J=1.8,2.6,7.4 \mathrm{~Hz}), 5.15(1 \mathrm{H}, \mathrm{dd}, J=5.3,5.2 \mathrm{~Hz}), 5.03(1 \mathrm{H}, \mathrm{s}), 3.89(3 \mathrm{H}, \mathrm{s}), 3.56(1 \mathrm{H}$, broad s), $3.07(1 \mathrm{H}, \mathrm{dd}, J=2.7,17.7 \mathrm{~Hz}), 3.01(1 \mathrm{H}, \mathrm{dd}, J=7.7,17.7 \mathrm{~Hz}), 2.80(1 \mathrm{H}, \mathrm{d}, J=$ $18.2 \mathrm{~Hz}), 2.69(1 \mathrm{H}$, dddd, $J=2.0,3.2,6.8,18.2 \mathrm{~Hz}) ;{ }^{13} \mathbf{C}-\mathbf{N M R}\left(100 \mathrm{MHz}, \mathrm{CDCl}_{3}\right) \delta$ $171.5(\mathrm{C}), 168.4(\mathrm{C}), 145.6(\mathrm{C}), 131.3(\mathrm{CH}), 129.6(\mathrm{CH}), 122.1(\mathrm{C}), 88.3(\mathrm{CH}), 85.3$ $(\mathrm{CH}), 59.0\left(\mathrm{CH}_{3}\right), 48.2(\mathrm{CH}), 39.7\left(\mathrm{CH}_{2}\right), 33.4\left(\mathrm{CH}_{2}\right)$; HRMS calcd for $\mathrm{C}_{12} \mathrm{H}_{13} \mathrm{O}_{4}$ $[\mathrm{M}+\mathrm{H}]^{+}$221.0814; found 221.0820

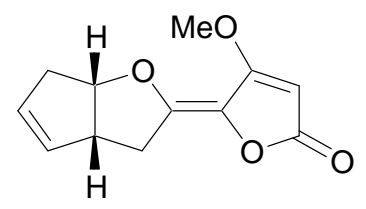

$\boldsymbol{E}$ - $\boldsymbol{\gamma}$-Ylidenetetronate $\boldsymbol{E}-\mathbf{2 4}: \boldsymbol{R}_{\mathbf{f}}=0.31$ (ethyl acetate/petroleum ether (1:1); UV detection); m.p. $=154-155{ }^{\circ} \mathrm{C}$, IR (KBr) $1751,1689,1584,1112,844 \mathrm{~cm}^{-1} ;{ }^{\mathbf{1}} \mathbf{H}-\mathbf{N M R}$ 
$\left(400 \mathrm{MHz}, \mathrm{CDCl}_{3}\right) \delta 5.74(1 \mathrm{H}$, ddd, $J=2.2,2.2,7.6 \mathrm{~Hz}), 5.57(1 \mathrm{H}, \mathrm{ddd}, J=2.0,3.9,5.8$ $\mathrm{Hz}), 5.23(1 \mathrm{H}, \mathrm{dd}, J=4.9,5.0 \mathrm{~Hz}), 5.06(1 \mathrm{H}, \mathrm{s}), 3.90(3 \mathrm{H}, \mathrm{s}), 3.50(1 \mathrm{H}, \mathrm{dddd}, J=1.5$, 3.3, 5.4, $12.7 \mathrm{~Hz}), 3.11(1 \mathrm{H}, \mathrm{dd}, J=7.4,18.0 \mathrm{~Hz}), 3.05(1 \mathrm{H}, \mathrm{dd}, J=3.2,18.0 \mathrm{~Hz}), 2.77$ $(1 \mathrm{H}, \mathrm{m}), 2.70(1 \mathrm{H}$, dddd, $J=2.0,2.5,6.4,18.3 \mathrm{~Hz}) ;{ }^{13} \mathbf{C}-\mathbf{N M R}\left(100 \mathrm{MHz}, \mathrm{CDCl}_{3}\right) \delta$ $170.1(\mathrm{C}), 169.2(\mathrm{C}), 149.9(\mathrm{C}), 131.6(\mathrm{CH}), 129.5(\mathrm{CH}), 124.6(\mathrm{C}), 90.1(\mathrm{CH}), 86.4$ $(\mathrm{CH}), 59.3\left(\mathrm{CH}_{3}\right), 47.3(\mathrm{CH}), 40.1\left(\mathrm{CH}_{2}\right), 34.6\left(\mathrm{CH}_{2}\right) ; \mathbf{H R M S}$ calcd for $\mathrm{C}_{12} \mathrm{H}_{13} \mathrm{O}_{4}$ $[\mathrm{M}+\mathrm{H}]^{+}$221.0814; found 221.0810

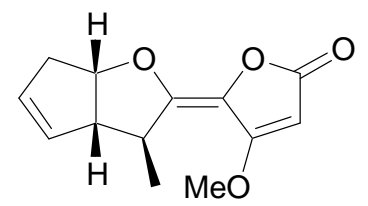

$\boldsymbol{Z}$ - $\boldsymbol{\gamma}$-Ylidenetetronate $\boldsymbol{Z}-\mathbf{2 5}: \boldsymbol{R}_{\mathbf{f}}=0.31$ (ethyl acetate/petroleum ether (1:1); UV detection); m.p. $=144-145^{\circ} \mathrm{C}$, IR (KBr) 3131, 2965, 2925, 1741, 1684, 1591, 1189, $1058,850,791 \mathrm{~cm}^{-1} ;{ }^{1} \mathbf{H}-\mathbf{N M R}\left(400 \mathrm{MHz}, \mathrm{CDCl}_{3}\right) \delta 5.73(1 \mathrm{H}, \mathrm{ddd}, J=0.6,2.0,5.2 \mathrm{~Hz})$, $5.54(1 \mathrm{H}, \mathrm{m}), 5.21(1 \mathrm{H}, \mathrm{dd}, J=4.9,5.1 \mathrm{~Hz}), 5.03(1 \mathrm{H}, \mathrm{s}), 3.90(3 \mathrm{H}, \mathrm{s}), 3.34(1 \mathrm{H}, \mathrm{q}, J=$ $7.2 \mathrm{~Hz}), 3.16(1 \mathrm{H}$, broad s), $2.76(1 \mathrm{H}, \mathrm{d}, J=18.2 \mathrm{~Hz}), 2.65(1 \mathrm{H}, \mathrm{dddd}, J=1.7,2.8,6.5$, $18.2 \mathrm{~Hz}), 1.27(3 \mathrm{H}, \mathrm{d}, J=7.2 \mathrm{~Hz}){ }^{13} \mathbf{C}-\mathrm{NMR}\left(100 \mathrm{MHz}, \mathrm{CDCl}_{3}\right) \delta 171.2(\mathrm{C}), 168.5(\mathrm{C})$, $151.1(\mathrm{C}), 130.9(\mathrm{CH}), 129.8(\mathrm{CH}), 122.2(\mathrm{C}), 86.6(\mathrm{CH}), 85.8(\mathrm{CH}), 59.1\left(\mathrm{CH}_{3}\right), 57.1$ $(\mathrm{CH}), 40.7(\mathrm{CH}), 39.8\left(\mathrm{CH}_{2}\right), 20.7\left(\mathrm{CH}_{3}\right)$; HRMS calcd for $\mathrm{C}_{13} \mathrm{H}_{15} \mathrm{O}_{4}[\mathrm{M}+\mathrm{H}]^{+}$235.0970; found 235.0969

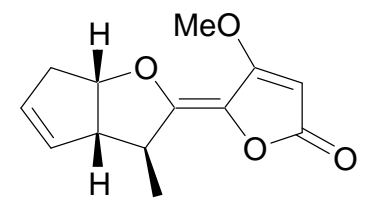

$\boldsymbol{E}$ - $\gamma$-Ylidenetetronate $\boldsymbol{E}$-25: $\boldsymbol{R}_{\mathbf{f}}=0.35$ (ethyl acetate/petroleum ether (1:1); UV detection); m.p. $=157-158{ }^{\circ} \mathrm{C}$, IR $(\mathrm{KBr}) 3124,2968,2931,1732,1680,1592,1176,831$, $787 \mathrm{~cm}^{-1} ;{ }^{1} \mathbf{H}$-NMR $\left(400 \mathrm{MHz}, \mathrm{CDCl}_{3}\right) \delta 5.74(1 \mathrm{H}, \mathrm{ddd}, J=2.4,4.6,7.8 \mathrm{~Hz}), 5.59(1 \mathrm{H}$, ddd, $J=1.8,2.4,7.4 \mathrm{~Hz}), 5.31(1 \mathrm{H}, \mathrm{dd}, J=4.8,4.8 \mathrm{~Hz}), 5.06(1 \mathrm{H}, \mathrm{s}), 3.91(3 \mathrm{H}, \mathrm{s}), 3.39$ $(1 \mathrm{H}, \mathrm{q}, J=7.4 \mathrm{~Hz}), 3.12(1 \mathrm{H}$, broad s), $2.75(1 \mathrm{H}, \mathrm{d}, 17.8 \mathrm{~Hz}), 2.68(1 \mathrm{H}, \mathrm{m}), 1.35(3 \mathrm{H}, \mathrm{d}$, $J=7.4 \mathrm{~Hz}) ;{ }^{13} \mathrm{C}-\mathrm{NMR}\left(100 \mathrm{MHz}, \mathrm{CDCl}_{3}\right) \delta 170.3(\mathrm{C}), 169.2(\mathrm{C}), 154.8(\mathrm{C}), 131.2(\mathrm{CH})$, $129.4(\mathrm{CH}), 124.3(\mathrm{C}), 88.5(\mathrm{CH}), 86.3(\mathrm{CH}), 59.3\left(\mathrm{CH}_{3}\right), 56.1(\mathrm{CH}), 42.2(\mathrm{CH}), 40.0$ $\left(\mathrm{CH}_{2}\right), 19.5\left(\mathrm{CH}_{3}\right) ; \mathbf{H R M S}$ calcd for $\mathrm{C}_{13} \mathrm{H}_{15} \mathrm{O}_{4}[\mathrm{M}+\mathrm{H}]^{+} 235.0970$; found 235.0970 


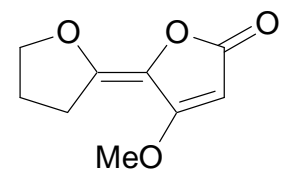

$\boldsymbol{Z}$ - $\boldsymbol{\gamma}$-Ylidenetetronate $\boldsymbol{Z}$-26: $\boldsymbol{R}_{\mathbf{f}}=0.22$ (ethyl acetate/petroleum ether (3:2); UV detection); m.p. $=119-120{ }^{\circ} \mathrm{C}$; IR (KBr) 3103, 2939, 1720, 1695, 1575, 1194, 1082, $1067,904,784 \mathrm{~cm}^{-1} ;{ }^{1} \mathbf{H}-\mathbf{N M R}\left(400 \mathrm{MHz}, \mathrm{CDCl}_{3}\right) \delta 5.06(1 \mathrm{H}, \mathrm{s}), 4.34(2 \mathrm{H}, \mathrm{dd}, J=6.9$, $7.0 \mathrm{~Hz}), 3.91(3 \mathrm{H}, \mathrm{s}), 2.93(2 \mathrm{H}, \mathrm{dd}, J=7.6,7.9 \mathrm{~Hz}), 2.17(1 \mathrm{H}, \mathrm{dd}, J=7.0,7.1 \mathrm{~Hz}), 2.14$ $(1 \mathrm{H}, \mathrm{dd}, J=7.5,7.5 \mathrm{~Hz}) ;{ }^{13} \mathrm{C}-\mathrm{NMR}\left(90 \mathrm{MHz}, \mathrm{CDCl}_{3}\right) \delta 171.5(\mathrm{C}), 168.6(\mathrm{C}), 145.9(\mathrm{C})$, $122.7(\mathrm{C}), 85.7(\mathrm{CH}), 73.0\left(\mathrm{CH}_{2}\right), 59.1\left(\mathrm{CH}_{3}\right), 28.2\left(\mathrm{CH}_{2}\right), 24.5\left(\mathrm{CH}_{2}\right)$; HRMS calcd for $\mathrm{C}_{9} \mathrm{H}_{11} \mathrm{O}_{4}[\mathrm{M}+\mathrm{H}]^{+}$183.0657; found 183.0650

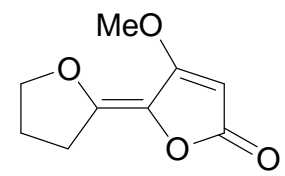

$\boldsymbol{E}$ - $\boldsymbol{\gamma}$-Ylidenetetronate $\boldsymbol{E}$-26: $\boldsymbol{R}_{\mathbf{f}}=0.28$ (ethyl acetate/petroleum ether (3:2); UV detection); m.p. $=78-80{ }^{\circ} \mathrm{C} ; \mathbf{I R}(\mathrm{KBr}) 1718,1695,1579,1410,1177,779 \mathrm{~cm}^{-1} ;{ }^{1} \mathbf{H}-$ NMR $\left(360 \mathrm{MHz}, \mathrm{CDCl}_{3}\right) \delta 5.10(1 \mathrm{H}, \mathrm{s}), 4.40(2 \mathrm{H}, \mathrm{dd}, J=6.9,7.1 \mathrm{~Hz}), 3.93(3 \mathrm{H}, \mathrm{s}), 2.96$ $(2 \mathrm{H}, \mathrm{dd}, J=7.6,7.6 \mathrm{~Hz}), 2.14(1 \mathrm{H}, \mathrm{dd}, J=7.2,7.2 \mathrm{~Hz}), 2.10(1 \mathrm{H}, \mathrm{dd}, J=7.4,7.4 \mathrm{~Hz})$; ${ }^{13}$ C-NMR $\left(100 \mathrm{MHz}, \mathrm{CDCl}_{3}\right) \delta 170.1(\mathrm{C}), 169.1(\mathrm{C}), 150.1(\mathrm{C}), 124.9(\mathrm{C}), 86.7(\mathrm{CH})$, $74.5\left(\mathrm{CH}_{2}\right), 59.4\left(\mathrm{CH}_{3}\right), 29.2\left(\mathrm{CH}_{2}\right), 23.6\left(\mathrm{CH}_{2}\right)$; HRMS calcd for $\mathrm{C}_{9} \mathrm{H}_{11} \mathrm{O}_{4}[\mathrm{M}+\mathrm{H}]^{+}$ 183.0657; found 183.0659<smiles>CC1CCOC1=C1OC(=O)C=C1[N+](=O)[O-]</smiles>

$\boldsymbol{Z}$ - $\gamma$-Ylidenetetronate $\boldsymbol{Z}$-27: $\boldsymbol{R}_{\mathbf{f}}=0.21$ (ethyl acetate/petroleum ether (1:1); UV detection), 0.28 (ethyl acetate/petroleum ether (3:2); UV detection); IR (KBr) 2978, 1732, 1689, 1586, 1208, 1184, 1061, $881 \mathrm{~cm}^{-1} ;{ }^{1} \mathbf{H}-\mathbf{N M R}\left(360 \mathrm{MHz}, \mathrm{CDCl}_{3}\right) \delta 5.07(1 \mathrm{H}$, s), $4.45(1 \mathrm{H}, \mathrm{dd}, J=8.9,9.0 \mathrm{~Hz}), 4.30(1 \mathrm{H}, \mathrm{ddd}, J=5.8,8.9,11.7 \mathrm{~Hz}), 3.92(3 \mathrm{H}, \mathrm{s}), 3.40$ $(1 \mathrm{H}, \mathrm{dq}, J=0.7,7.4 \mathrm{~Hz}), 2.26(1 \mathrm{H}$, ddd, $J=8.0,12.1,20.3 \mathrm{~Hz}), 1.87(1 \mathrm{H}, \mathrm{ddd}, J=0.9$, 5.7, $12.4 \mathrm{~Hz}), 1.21(3 \mathrm{H}, \mathrm{d}, J=7.3 \mathrm{~Hz}) ;{ }^{13} \mathrm{C}-\mathrm{NMR}\left(100 \mathrm{MHz}, \mathrm{CDCl}_{3}\right) \delta 171.2(\mathrm{C}), 168.5$ $(\mathrm{C}), 151.2(\mathrm{C}), 122.7(\mathrm{C}), 85.9(\mathrm{CH}), 71.1\left(\mathrm{CH}_{2}\right), 59.2\left(\mathrm{CH}_{3}\right), 35.0(\mathrm{CH}), 32.4\left(\mathrm{CH}_{2}\right)$, $19.1\left(\mathrm{CH}_{3}\right) ;$ HRMS calcd for $\mathrm{C}_{10} \mathrm{H}_{13} \mathrm{O}_{4}[\mathrm{M}+\mathrm{H}]^{+}$197.0814; found 197.0813 


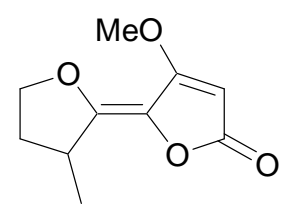

$\boldsymbol{E}$ - $\gamma$-Ylidenetetronate $\boldsymbol{E}$-27: $\boldsymbol{R}_{\mathbf{f}}=0.23$ (ethyl acetate/petroleum ether (1:1); UV detection), 0.31 (ethyl acetate/petroleum ether (3:2), UV detection); IR (KBr) 3116, 2980, 2938, 1737, 1689, 1583, 1443, 1408, 1233, 1174, 885, $778 \mathrm{~cm}^{-1} ;{ }^{1} \mathbf{H}-\mathbf{N M R}(360$ $\left.\mathrm{MHz}, \mathrm{CDCl}_{3}\right) \delta 5.08(1 \mathrm{H}, \mathrm{s}), 4.40(2 \mathrm{H}, \mathrm{m}), 3.92(3 \mathrm{H}, \mathrm{s}), 3.38(1 \mathrm{H}, \mathrm{dq}, J=3.5,7.3 \mathrm{~Hz})$, $2.26(1 \mathrm{H}, \mathrm{ddd}, J=8.4,12.7,17.2 \mathrm{~Hz}), 1.80(1 \mathrm{H}$, dddd, $J=3.5,4.3,6.3,12.6 \mathrm{~Hz}), 1.31$ $(3 \mathrm{H}, \mathrm{d}, J=7.3 \mathrm{~Hz}) ;{ }^{13} \mathbf{C}-\mathbf{N M R}\left(100 \mathrm{MHz}, \mathrm{CDCl}_{3}\right) \delta 170.4(\mathrm{C}), 169.0(\mathrm{C}), 154.4(\mathrm{C})$, $124.9(\mathrm{C}), 86.5(\mathrm{CH}), 72.8\left(\mathrm{CH}_{2}\right), 59.4\left(\mathrm{CH}_{3}\right), 36.8(\mathrm{CH}), 31.8\left(\mathrm{CH}_{2}\right), 17.8\left(\mathrm{CH}_{3}\right)$; HRMS calcd for $\mathrm{C}_{10} \mathrm{H}_{13} \mathrm{O}_{4}[\mathrm{M}+\mathrm{H}]^{+}$197.0814; found 197.0820

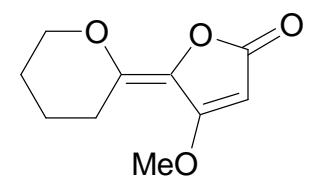

$\boldsymbol{Z}$ - $\gamma$-Ylidenetetronate $\boldsymbol{Z}-\mathbf{2 8}: \boldsymbol{R}_{\mathbf{f}}=0.25$ (ethyl acetate/petroleum ether (3:2); UV detection); m.p. $=90-91{ }^{\circ} \mathrm{C} ; \mathbf{I R}(\mathrm{KBr}) 3105,2943,1733,1668,1583,1213,1065,896$, $755 \mathrm{~cm}^{-1} ;{ }^{1} \mathbf{H}-\mathbf{N M R}\left(400 \mathrm{MHz}, \mathrm{CDCl}_{3}\right) \delta 5.13(1 \mathrm{H}, \mathrm{s}), 4.16(2 \mathrm{H}, \mathrm{dd}, J=5.4,5.6 \mathrm{~Hz})$, $3.89(3 \mathrm{H}, \mathrm{s}), 2.70(2 \mathrm{H}, \mathrm{dd}, J=6.3,6.7 \mathrm{~Hz}), 1.75-1.90(4 \mathrm{H}, \mathrm{m}) ;{ }^{13} \mathbf{C}-\mathbf{N M R}(100 \mathrm{MHz}$, $\left.\mathrm{CDCl}_{3}\right) \delta 171.4(\mathrm{C}), 167.9(\mathrm{C}), 143.9(\mathrm{C}), 127.7(\mathrm{C}), 87.6(\mathrm{CH}), 68.9\left(\mathrm{CH}_{2}\right), 59.1\left(\mathrm{CH}_{3}\right)$, $24.0\left(\mathrm{CH}_{2}\right), 23.3\left(\mathrm{CH}_{2}\right), 19.8\left(\mathrm{CH}_{2}\right)$; HRMS calcd for $\mathrm{C}_{10} \mathrm{H}_{13} \mathrm{O}_{4}[\mathrm{M}+\mathrm{H}]^{+} 197.0814$; found 197.0813

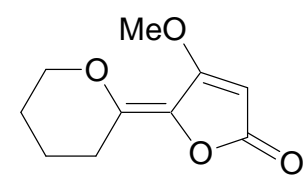

$\boldsymbol{E}$ - $\gamma$-Ylidenetetronate $\boldsymbol{E}$-28: $\boldsymbol{R}_{\mathbf{f}}=0.21$ (ethyl acetate/petroleum ether (1:1); UV detection), 0.30 (ethyl acetate/petroleum ether (3:2); UV detection); m.p. $=118-119{ }^{\circ} \mathrm{C}$, IR $(\mathrm{KBr}) 3118,2951,1743,1671,1584,1209,1106,1049,888 \mathrm{~cm}^{-1} ;{ }^{1} \mathbf{H}-\mathbf{N M R}(400$ $\left.\mathrm{MHz}, \mathrm{CDCl}_{3}\right) \delta 5.15(1 \mathrm{H}, \mathrm{s}), 4.10(2 \mathrm{H}, \mathrm{dd}, J=5.2,5.4 \mathrm{~Hz}), 3.91(3 \mathrm{H}, \mathrm{s}), 2.62(2 \mathrm{H}, \mathrm{dd}, J$ $=6.4,6.6 \mathrm{~Hz}), 1.76-1.89(4 \mathrm{H}, \mathrm{m}) ;{ }^{13} \mathrm{C}-\mathrm{NMR}\left(100 \mathrm{MHz}, \mathrm{CDCl}_{3}\right) \delta 170.7(\mathrm{C}), 168.6(\mathrm{C})$, 147.1 (C), $130.1(\mathrm{C}), 88.0(\mathrm{CH}), 69.8\left(\mathrm{CH}_{2}\right), 59.3\left(\mathrm{CH}_{3}\right), 24.3\left(\mathrm{CH}_{2}\right), 24.2\left(\mathrm{CH}_{2}\right), 20.1$ $\left(\mathrm{CH}_{2}\right)$; HRMS calcd for $\mathrm{C}_{10} \mathrm{H}_{13} \mathrm{O}_{4}[\mathrm{M}+\mathrm{H}]^{+}$197.0814; found 197.0822 


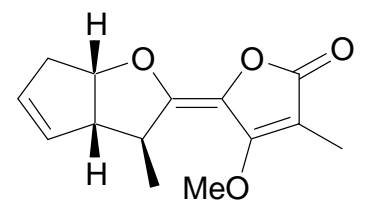

$\boldsymbol{Z}$ - $\boldsymbol{\gamma}$-Ylidenetetronate $\boldsymbol{Z}-29: \boldsymbol{R}_{\mathbf{f}}=0.22$ (ethyl acetate/petroleum ether (2:3); UV detection); IR (KBr) 2954, 1732, 1688, 1612, 1201, $1013 \mathrm{~cm}^{-1} ;{ }^{\mathbf{1}} \mathbf{H}-\mathbf{N M R}(400 \mathrm{MHz}$, $\left.\mathrm{CDCl}_{3}\right) \delta 5.71(1 \mathrm{H}, \mathrm{ddd}, J=2.0,2.8,5.0 \mathrm{~Hz}), 5.54(1 \mathrm{H}, \mathrm{m}), 5.16(1 \mathrm{H}, \mathrm{dd}, J=4.8,4.8$ $\mathrm{Hz}), 4.12(3 \mathrm{H}, \mathrm{s}), 3.32(1 \mathrm{H}, \mathrm{q}, J=7.2 \mathrm{~Hz}), 3.13(1 \mathrm{H}$, broad s), $2.74(1 \mathrm{H}, \mathrm{d}, J=18.2 \mathrm{~Hz})$, $2.63(1 \mathrm{H}, \mathrm{dddd}, J=1.8,2.8,6.5,18.2 \mathrm{~Hz}), 2.03(3 \mathrm{H}, \mathrm{s}), 1.26(3 \mathrm{H}, \mathrm{d}, J=7.2 \mathrm{~Hz}) ;{ }^{13} \mathrm{C}-$ NMR (100 MHz, CDCl $)_{3} \delta 170.4(\mathrm{C}), 163.8(\mathrm{C}), 149.6(\mathrm{C}), 131.0(\mathrm{CH}), 129.9(\mathrm{CH})$, $122.6(\mathrm{C}), 96.0(\mathrm{C}), 86.0(\mathrm{CH}), 59.1\left(\mathrm{CH}_{3}\right), 57.3(\mathrm{CH}), 40.6(\mathrm{CH}), 40.0\left(\mathrm{CH}_{2}\right), 20.9$ $\left(\mathrm{CH}_{3}\right), 9.3\left(\mathrm{CH}_{3}\right)$; HRMS calcd for $\mathrm{C}_{14} \mathrm{H}_{17} \mathrm{O}_{4}[\mathrm{M}+\mathrm{H}]^{+}$249.1127; found 249.1128

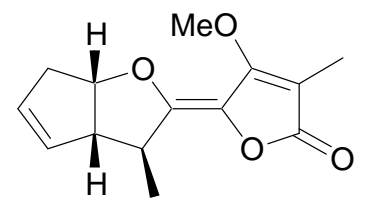

$\boldsymbol{E}$ - $\gamma$-Ylidenetetronate $\boldsymbol{E}$-29: $\boldsymbol{R}_{\mathbf{f}}=0.28$ (ethyl acetate/petroleum ether (2:3); UV detection); m.p. $=115^{\circ} \mathrm{C}$; IR (KBr) $1729,1683,1612,1405,1191,999,936 \mathrm{~cm}^{-1} ;{ }^{1} \mathbf{H}-$ NMR $\left(400 \mathrm{MHz}, \mathrm{CDCl}_{3}\right) \delta 5.73(1 \mathrm{H}, \mathrm{ddd}, J=1.8,2.1,7.6 \mathrm{~Hz}), 5.58(1 \mathrm{H}$, ddd, $J=1.8$, $3.6,5.4 \mathrm{~Hz}), 5.28(1 \mathrm{H}, \mathrm{dd}, J=4.6,4.6 \mathrm{~Hz}), 4.11(3 \mathrm{H}, \mathrm{s}), 3.80(1 \mathrm{H}, \mathrm{q}, J=7.2 \mathrm{~Hz}), 3.10$ $\left(1 \mathrm{H}\right.$, broad s), 2.62-2.77 $(2 \mathrm{H}, \mathrm{m}), 2.02(3 \mathrm{H}, \mathrm{s}), 1.33(3 \mathrm{H}, \mathrm{d}, J=7.2 \mathrm{~Hz}){ }^{13} \mathbf{C}-\mathbf{N M R}(100$ $\left.\mathrm{MHz}, \mathrm{CDCl}_{3}\right) \delta 171.1(\mathrm{C}), 163.4(\mathrm{C}), 153.1(\mathrm{C}), 131.3(\mathrm{CH}), 129.4(\mathrm{CH}), 124.4(\mathrm{C}), 97.9$ (C), $87.9(\mathrm{CH}), 59.5\left(\mathrm{CH}_{3}\right), 56.2(\mathrm{CH}), 42.0(\mathrm{CH}), 40.1\left(\mathrm{CH}_{2}\right), 19.7\left(\mathrm{CH}_{3}\right), 8.8\left(\mathrm{CH}_{3}\right)$; HRMS calcd for $\mathrm{C}_{14} \mathrm{H}_{17} \mathrm{O}_{4}[\mathrm{M}+\mathrm{H}]^{+} 249.1127$; found 249.1126 

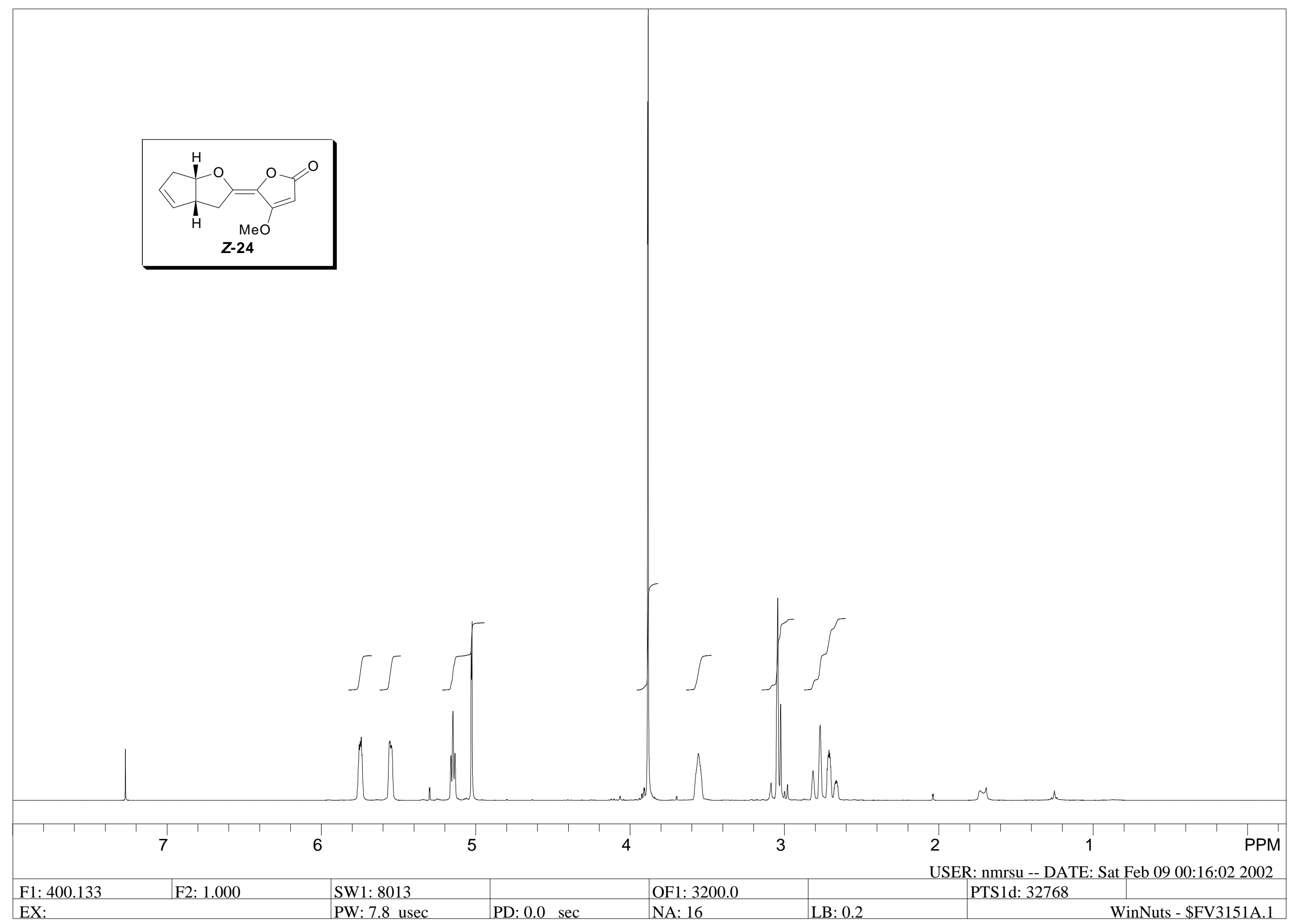


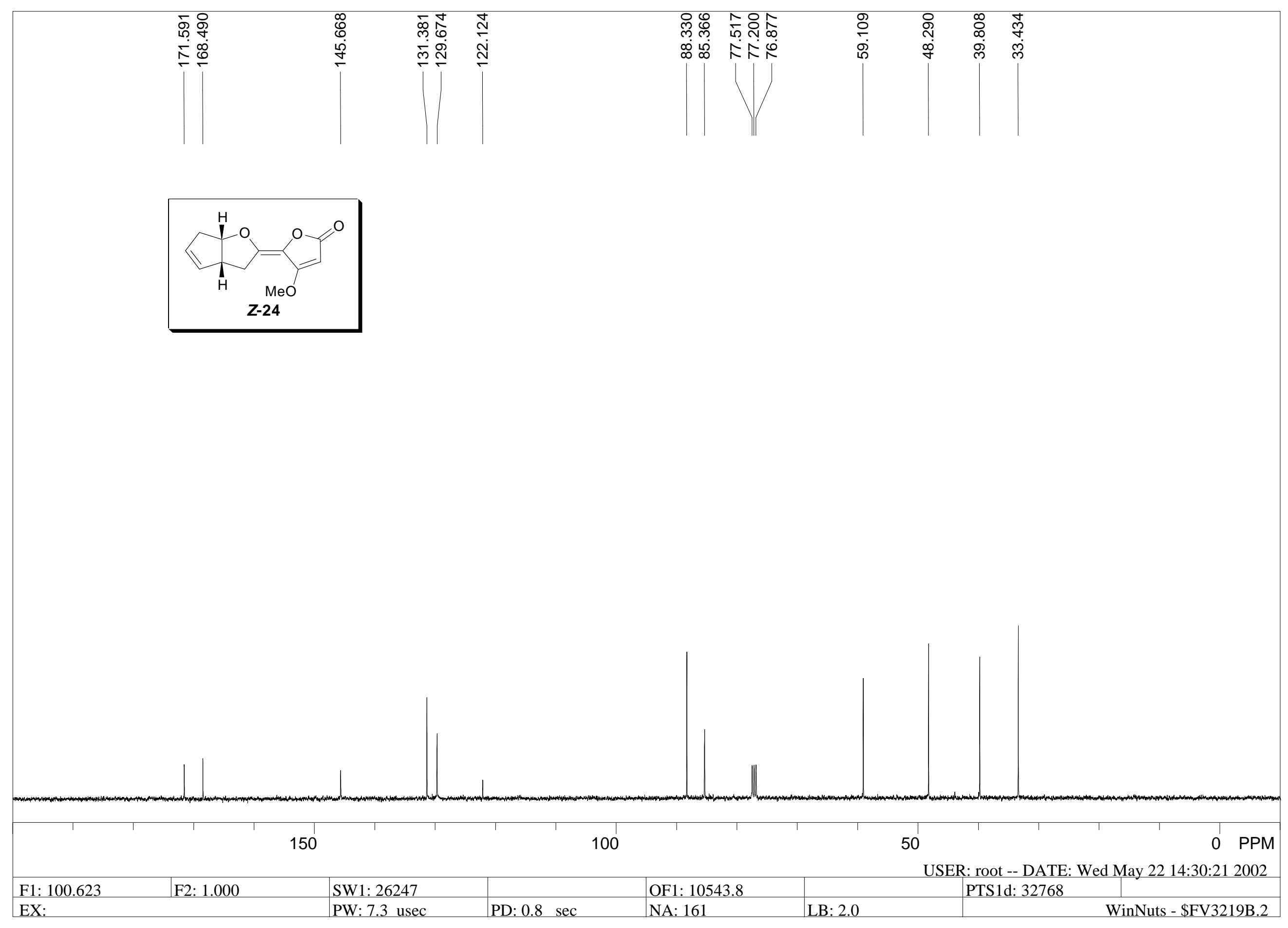




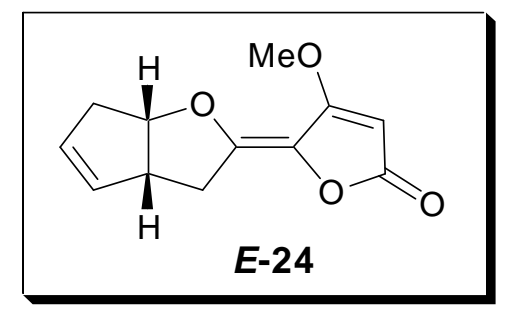




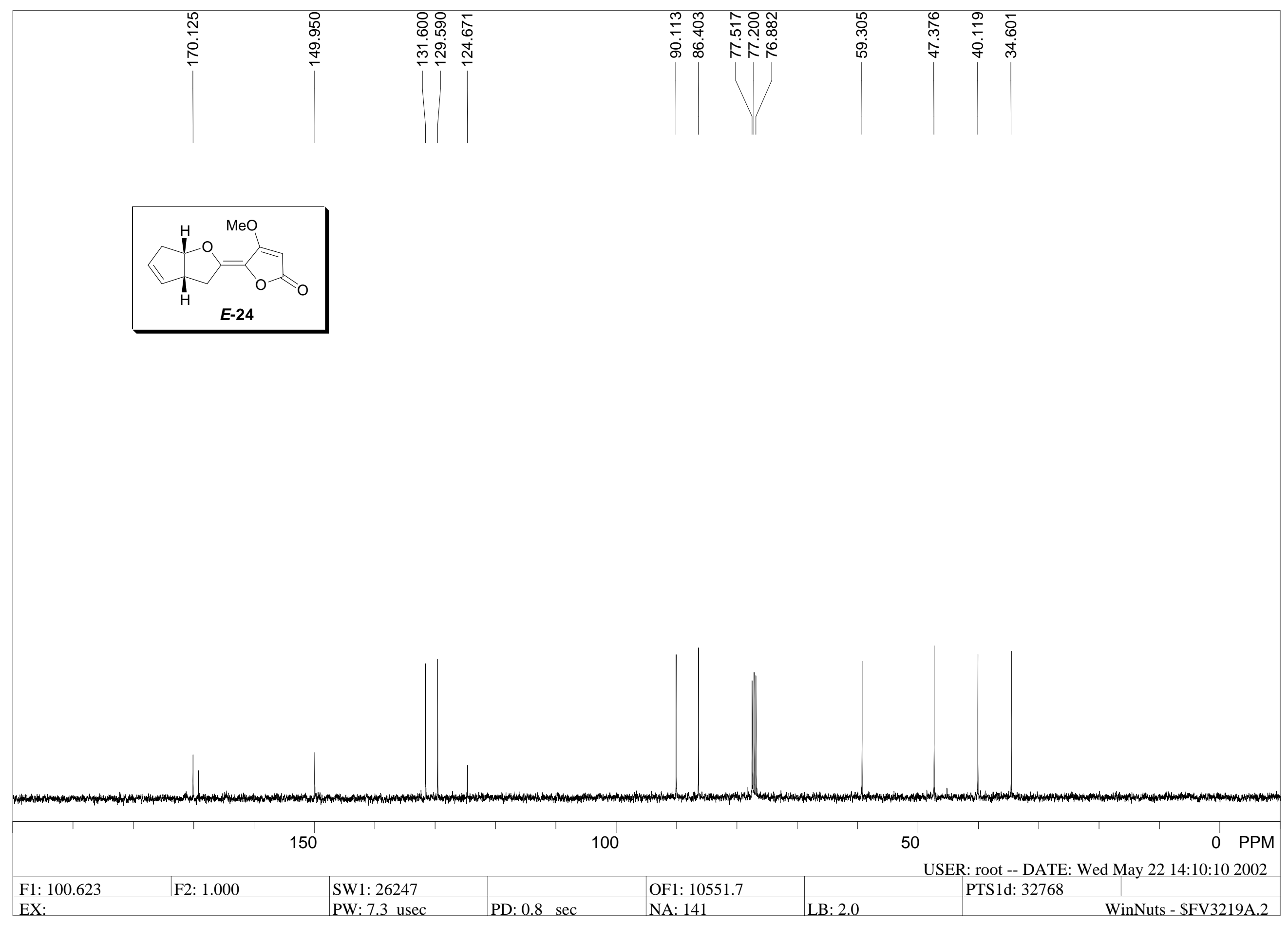



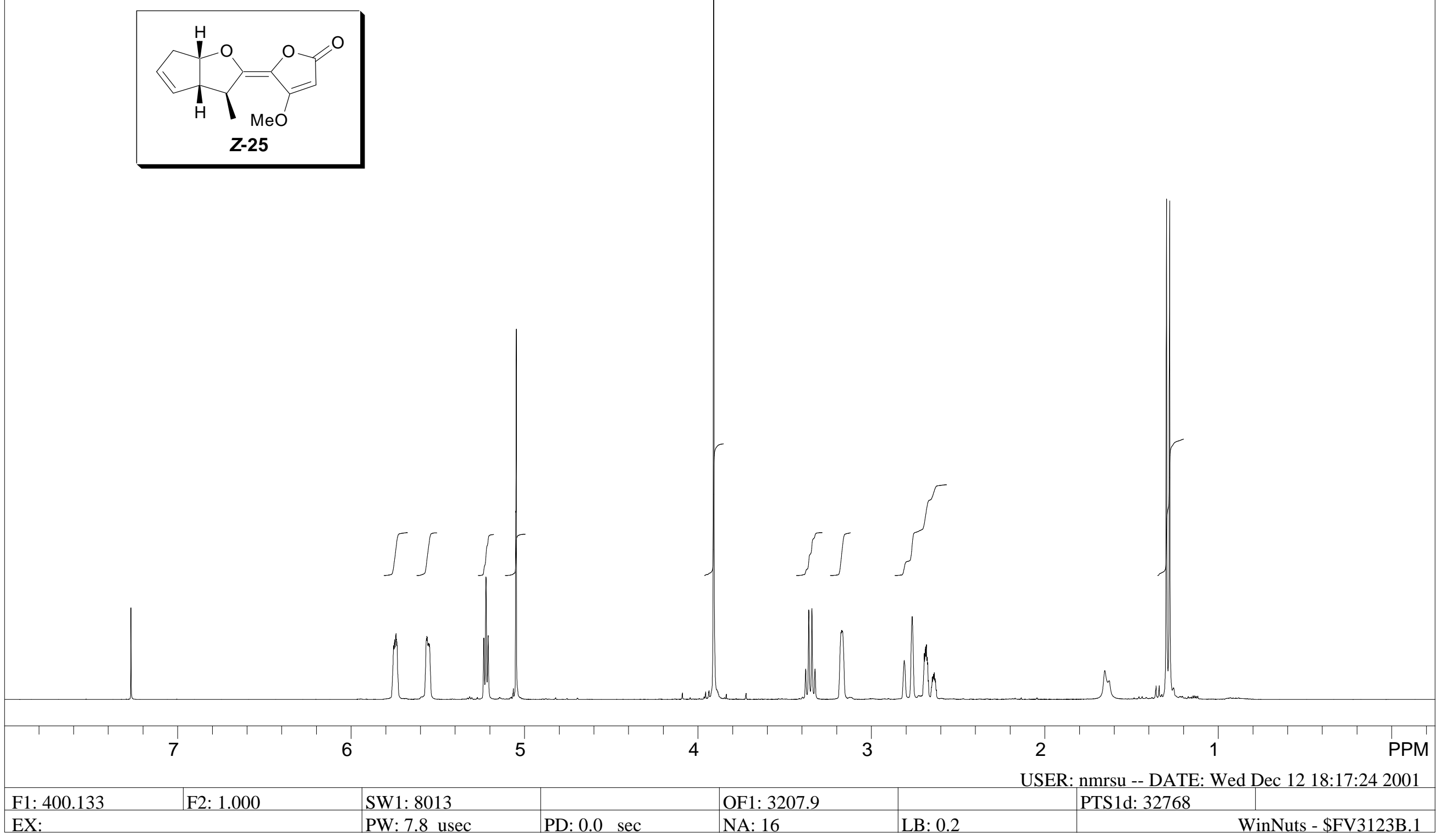


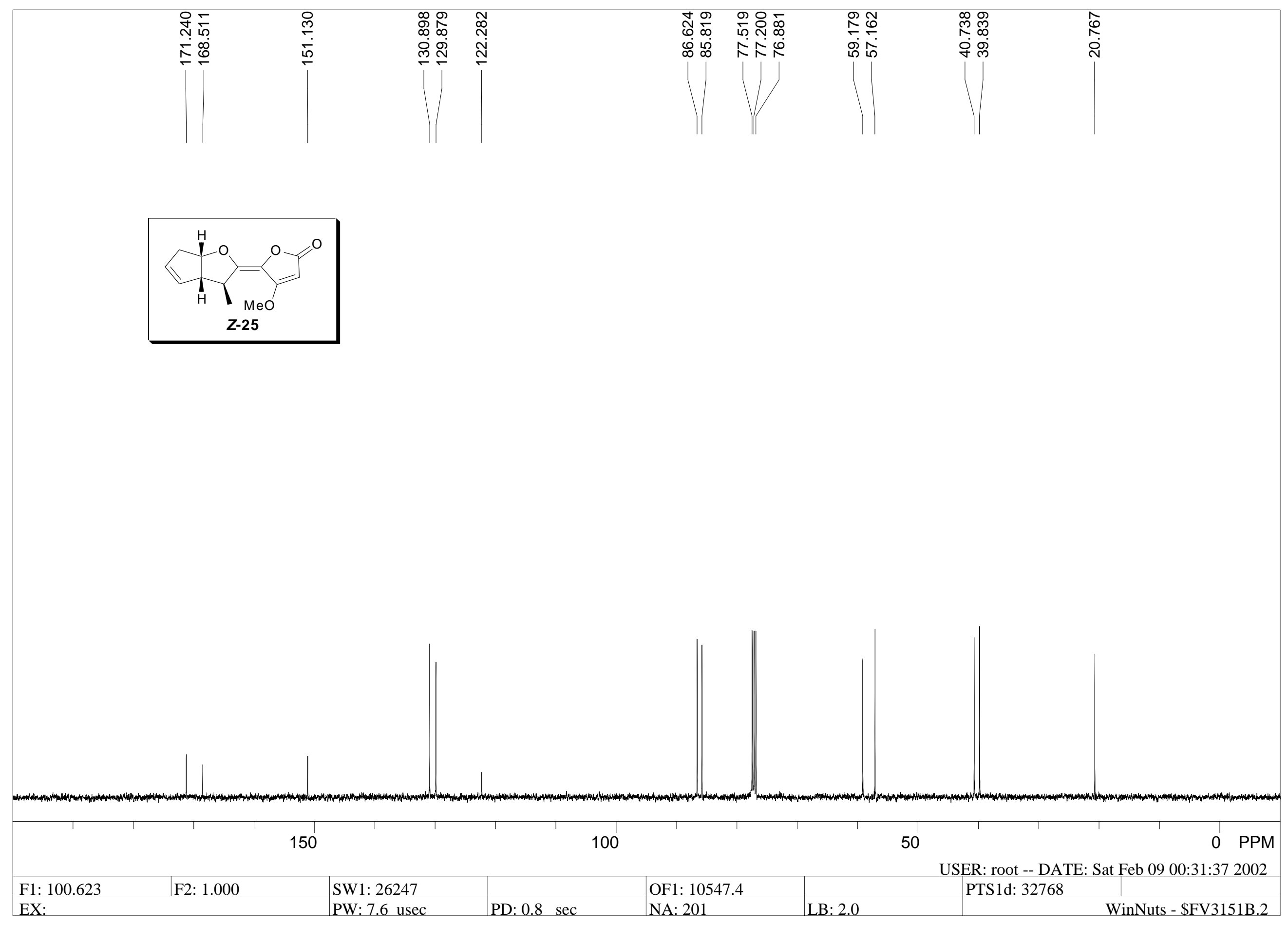




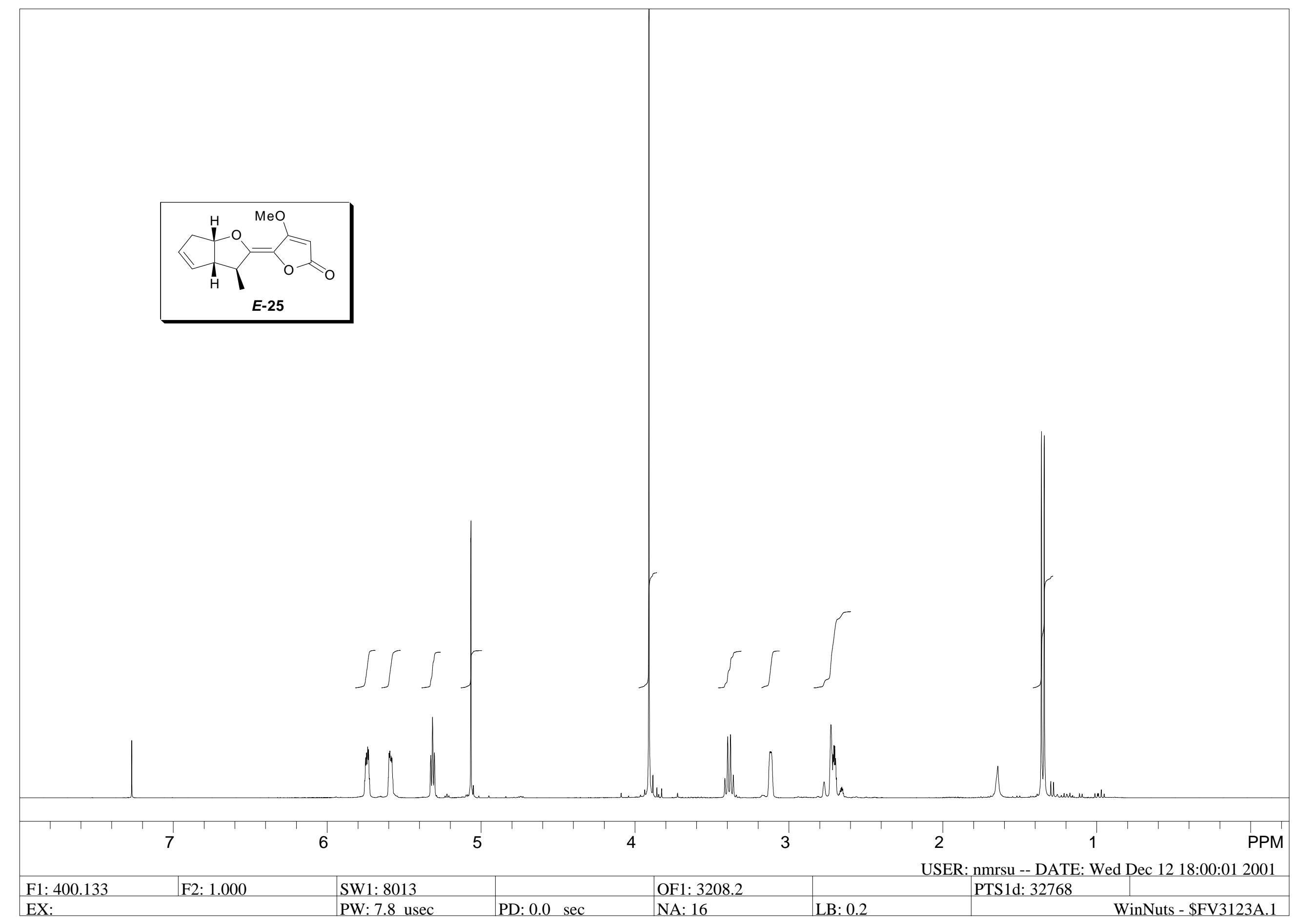




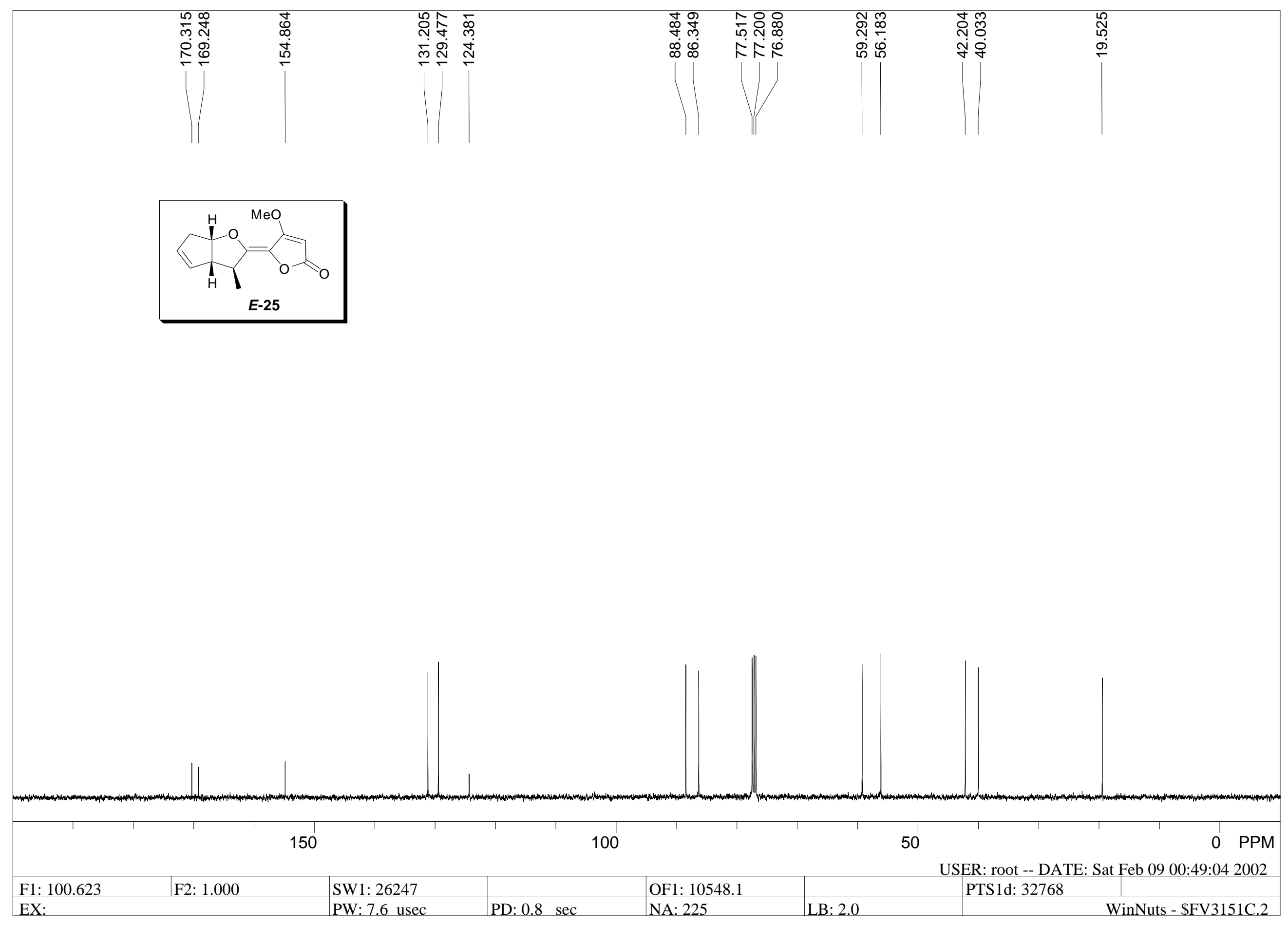




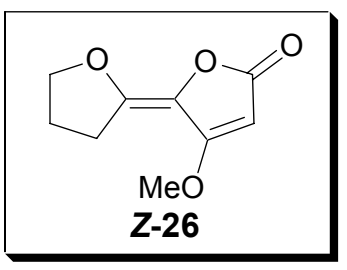

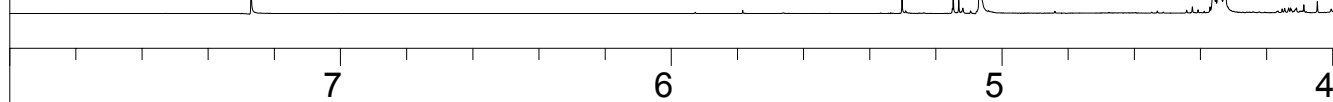

OF1: 3200.1

NA: 16
2 PTS1d: 32768 


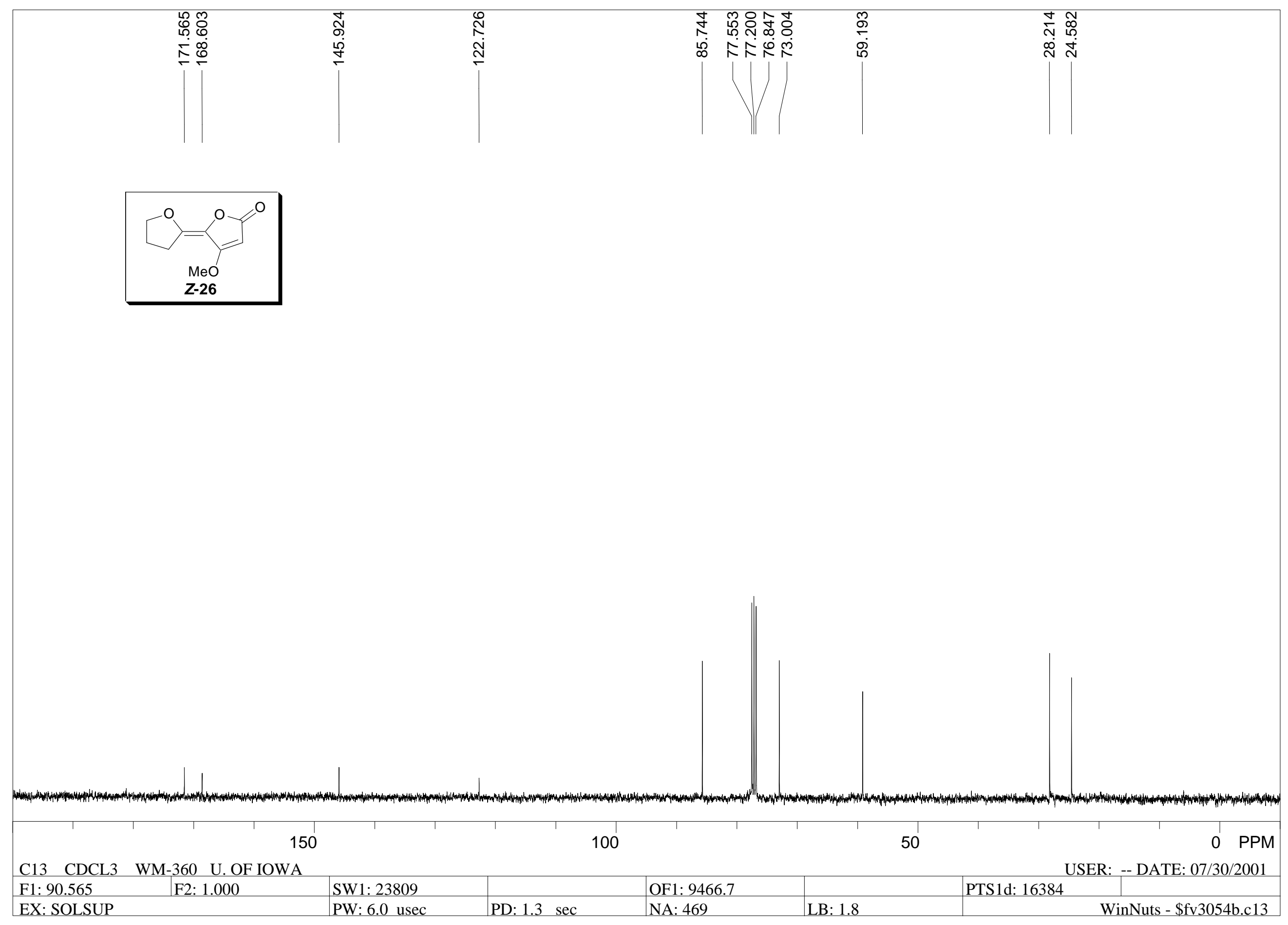



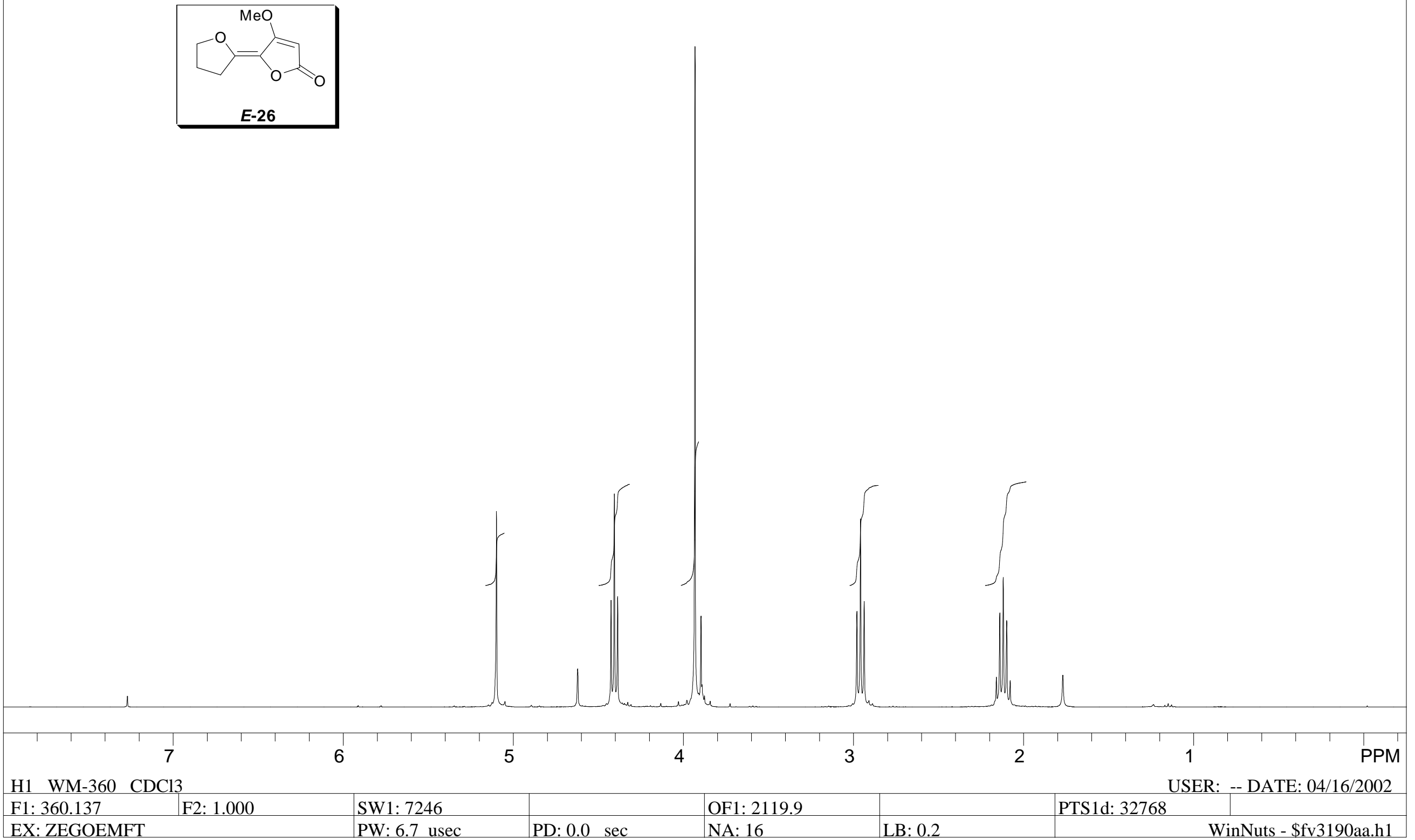


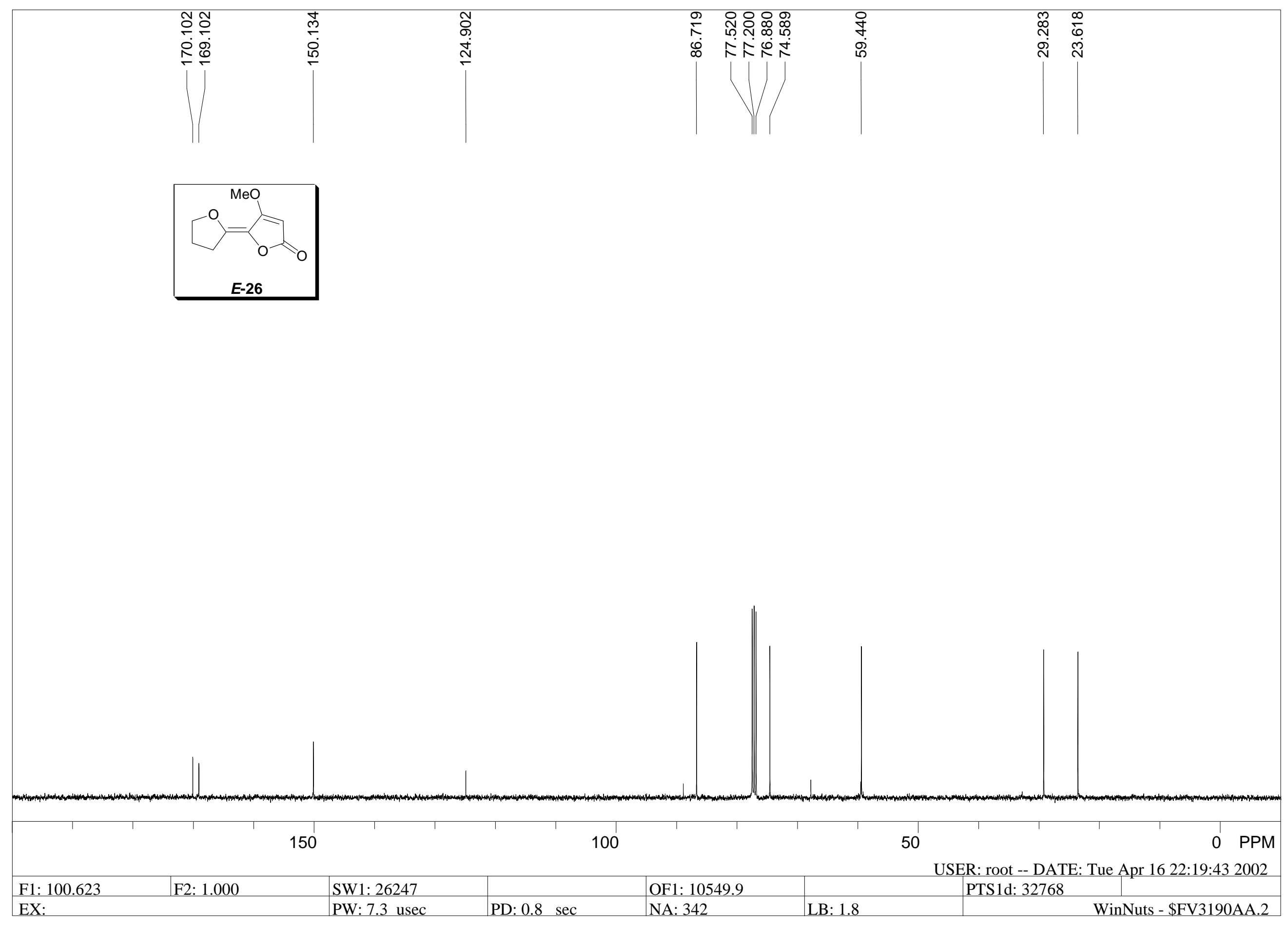




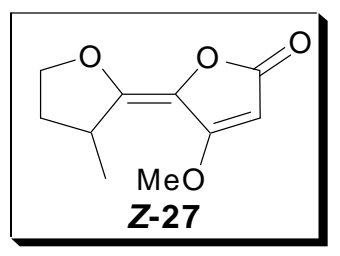

Z-27

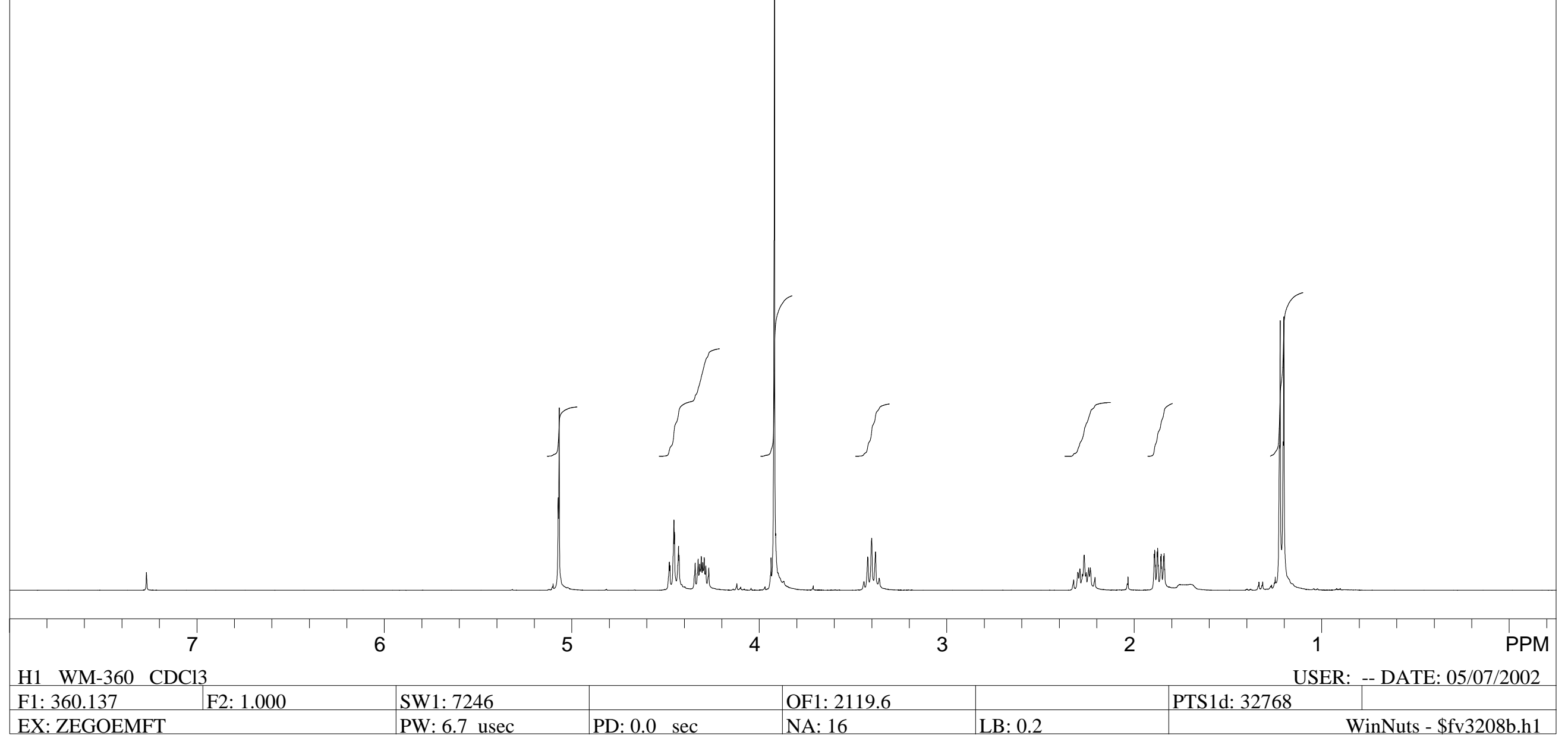




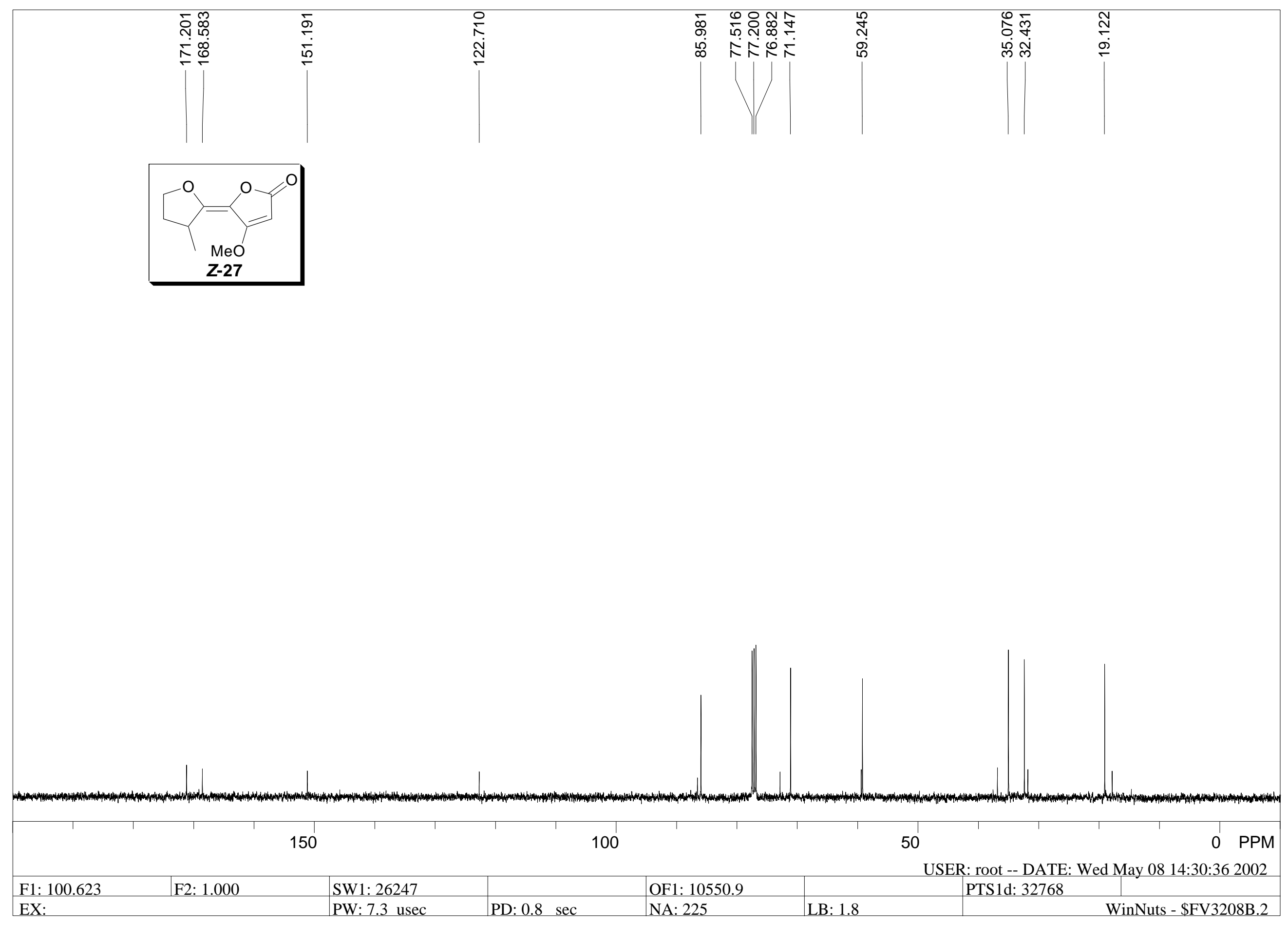



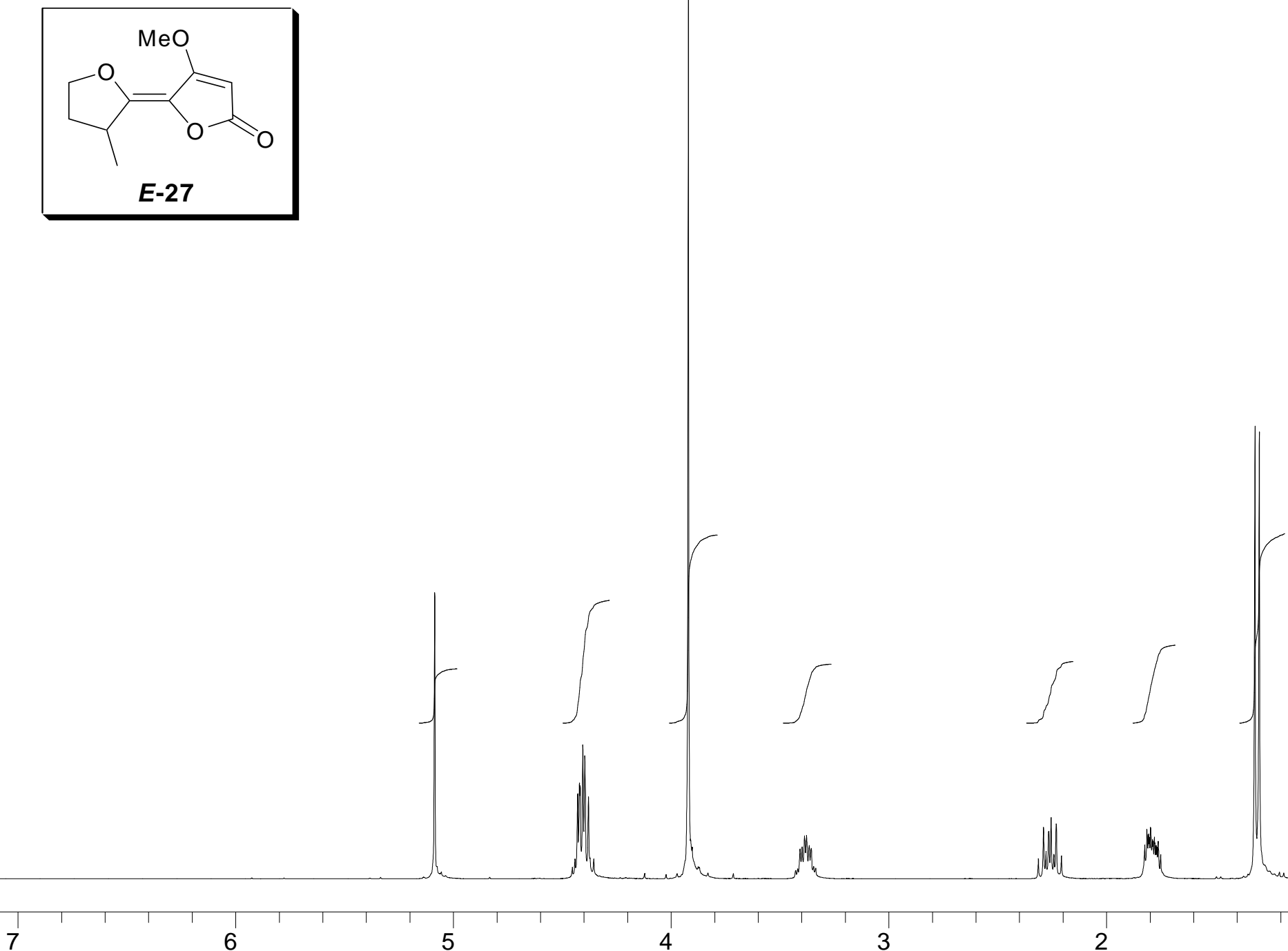

6

5

4

3

2

1

PPM

H1 WM-360 CDCl3

\begin{tabular}{l|l|l} 
F1: 360.137 & F2: 1.000 & SW1: 7246
\end{tabular}

OF1: 2119.9

USER: -- DATE: 05/07/2002

EX: ZEGOEMFT

PW: 6.7 usec

PD: $0.0 \mathrm{sec}$

NA: 16

LB: 0.2

PTS1d: 32768

WinNuts - \$fv3208ah1 


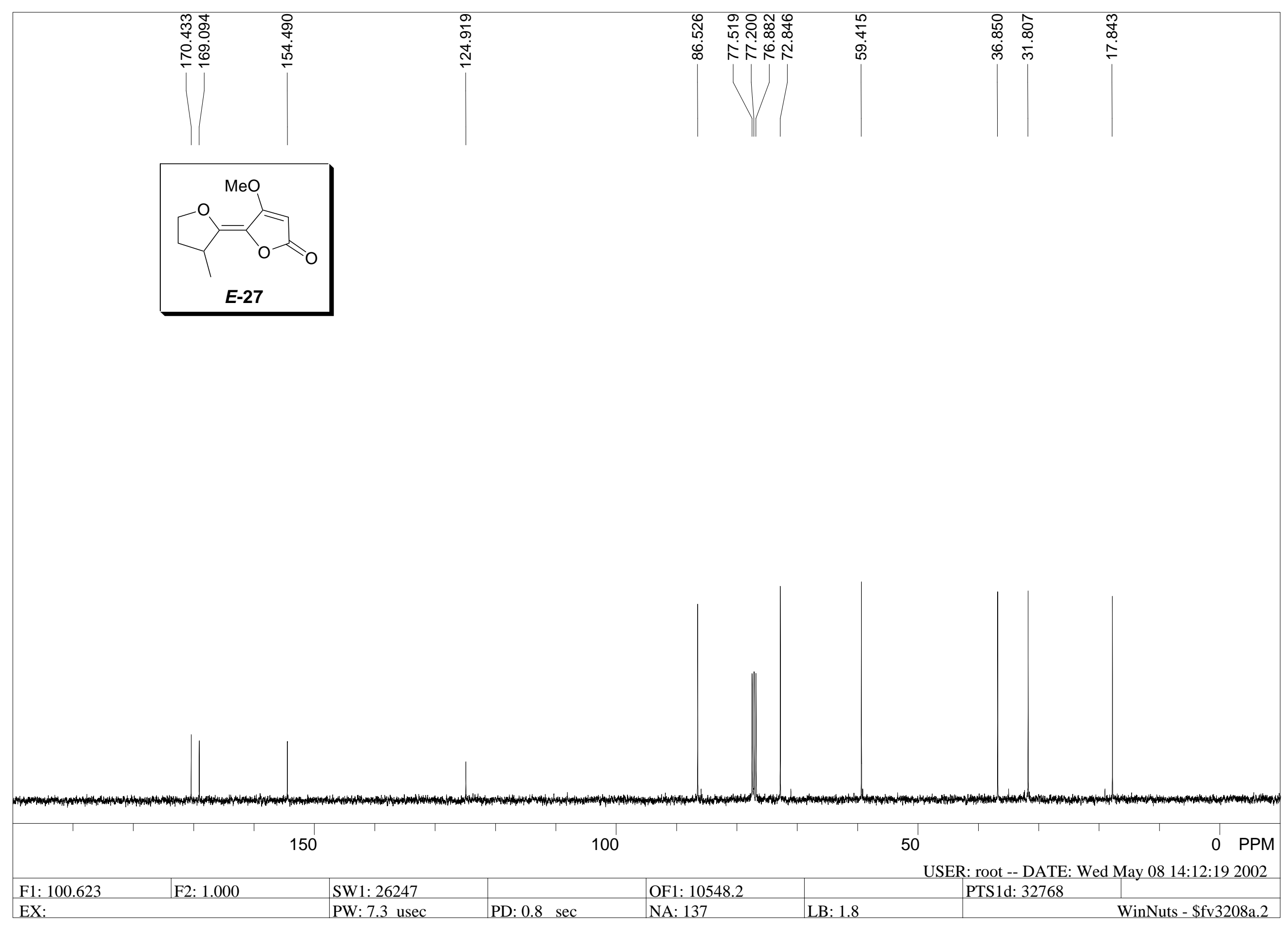



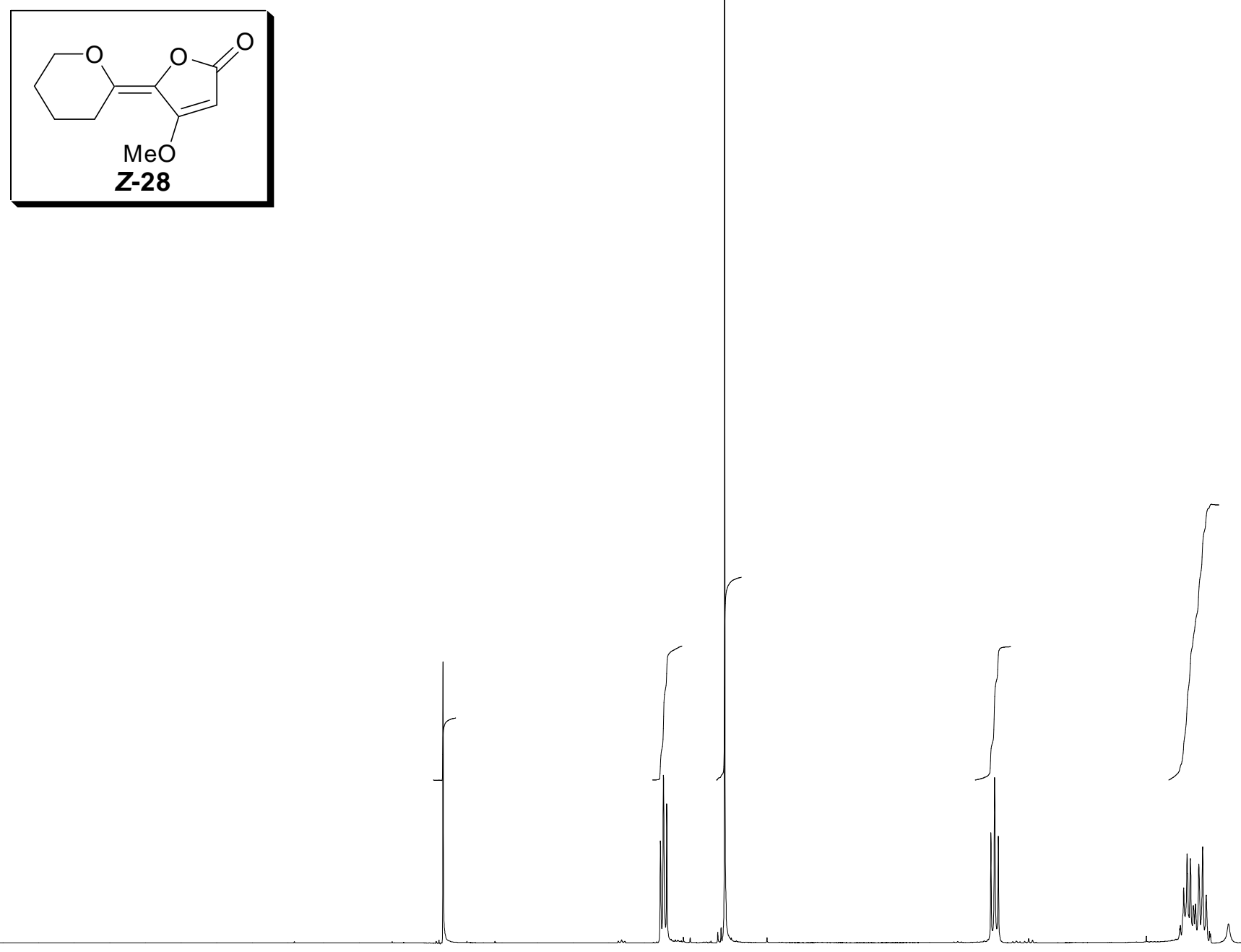

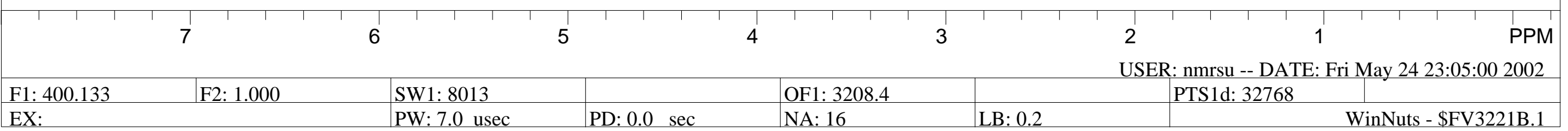



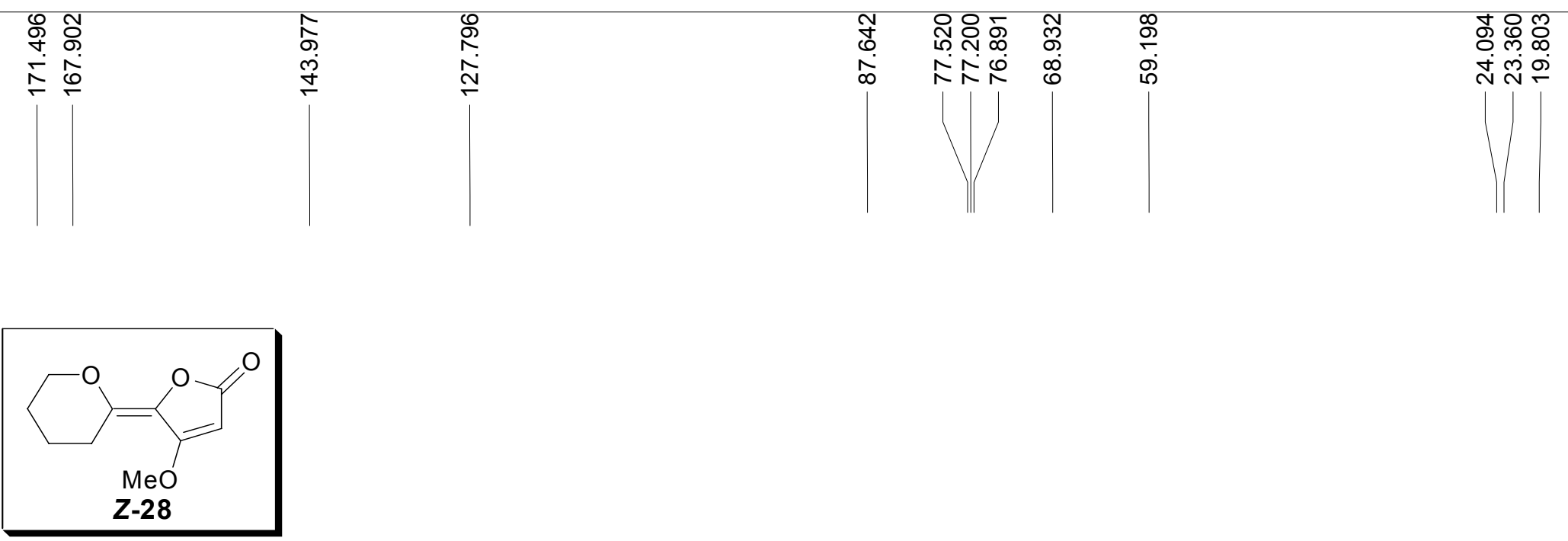

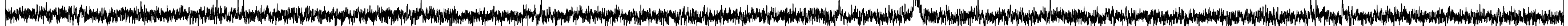

PD: $0.8 \mathrm{sec}$

OF1: 10554.4

NA: 181

USER: root -- DATE: Fri May 24 23:11:20 2002 PTS1d: 32768 


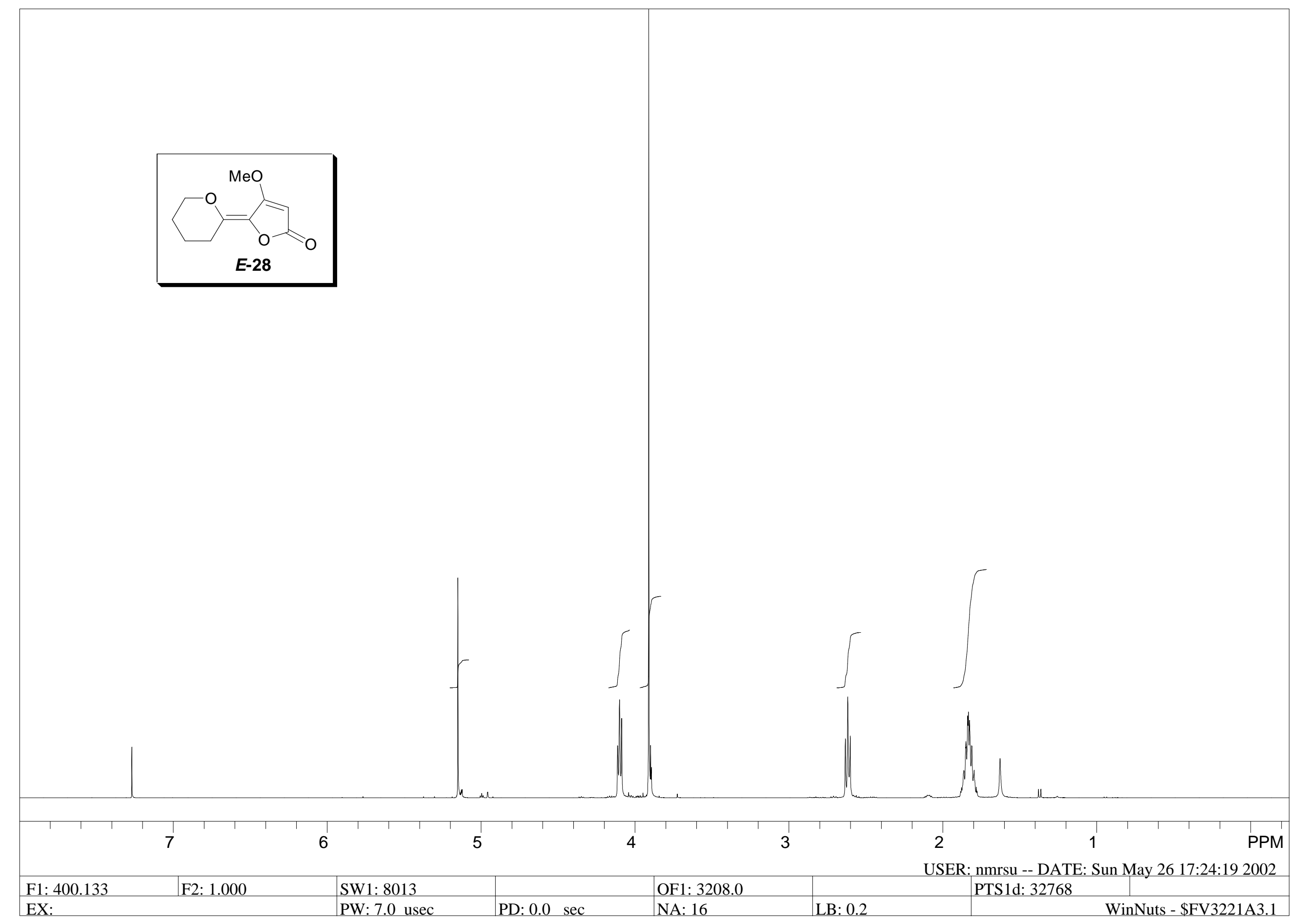




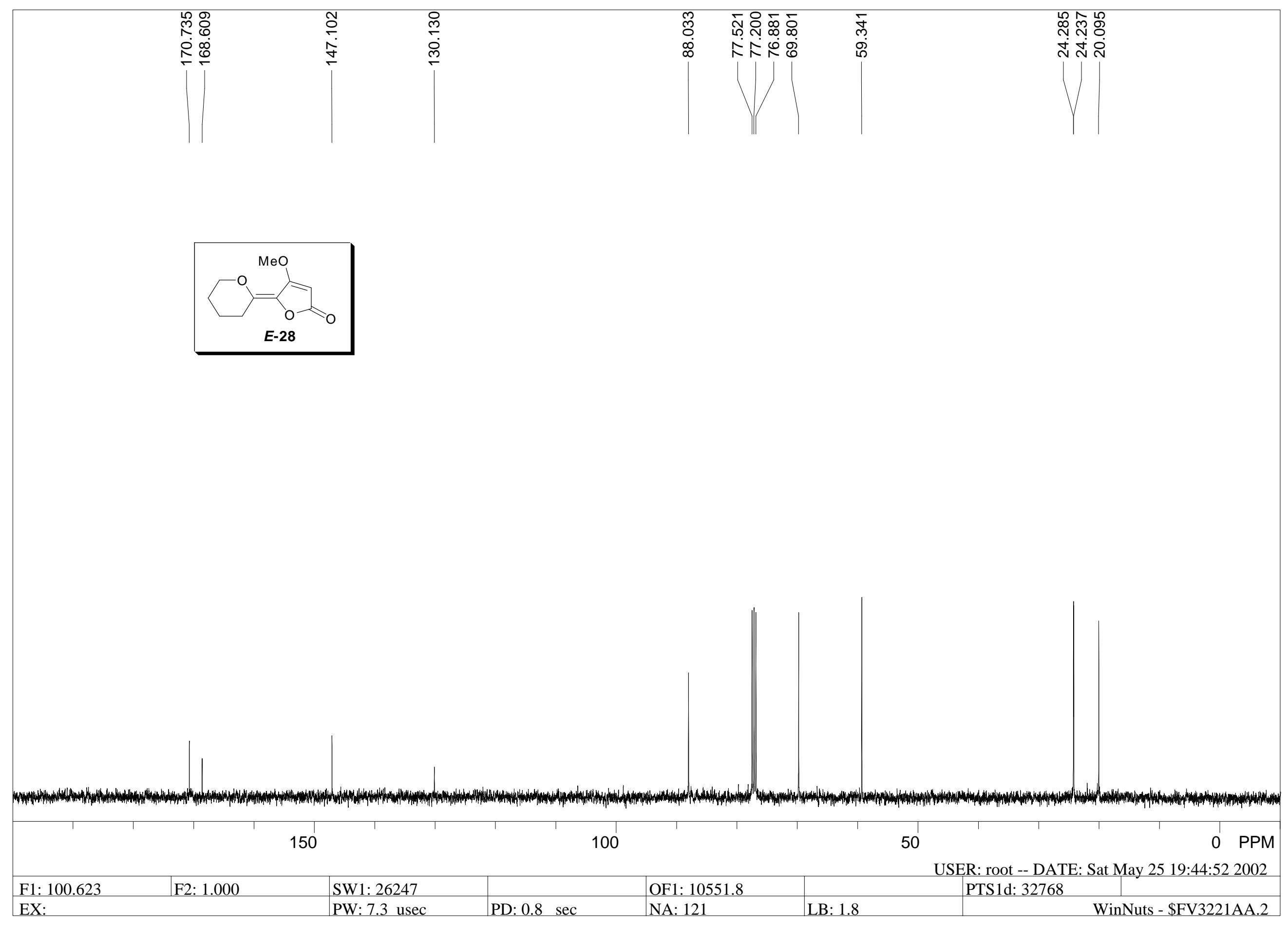




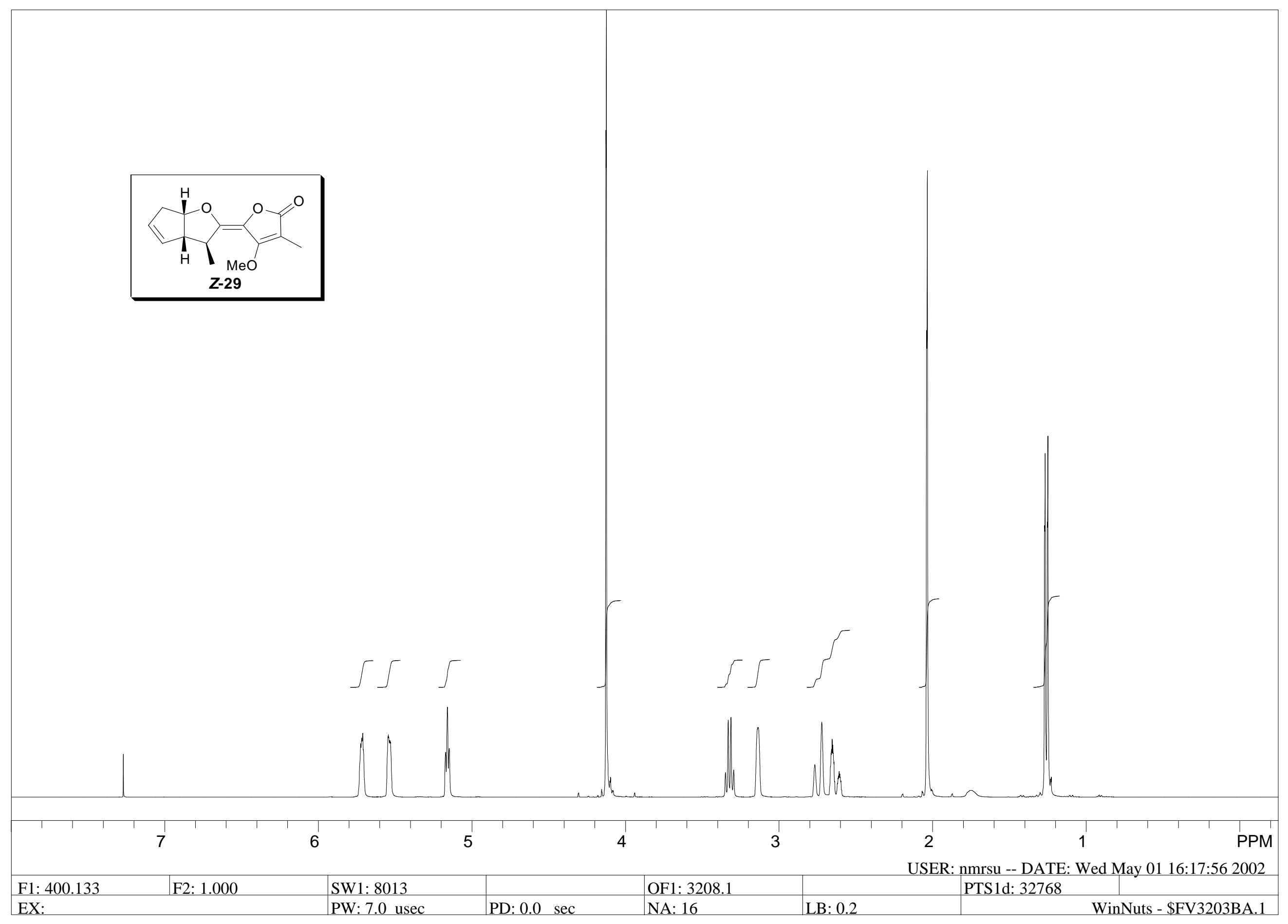




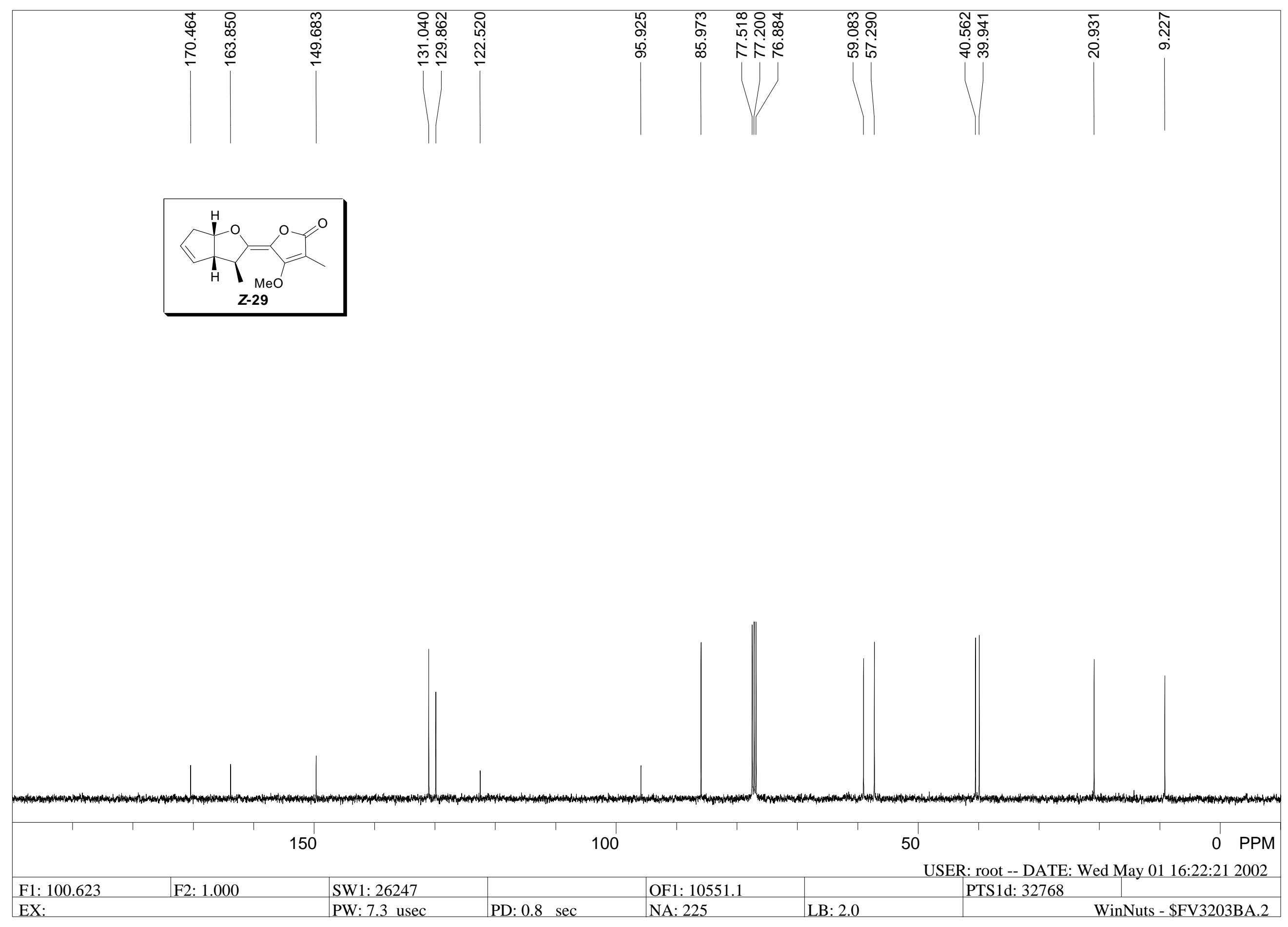



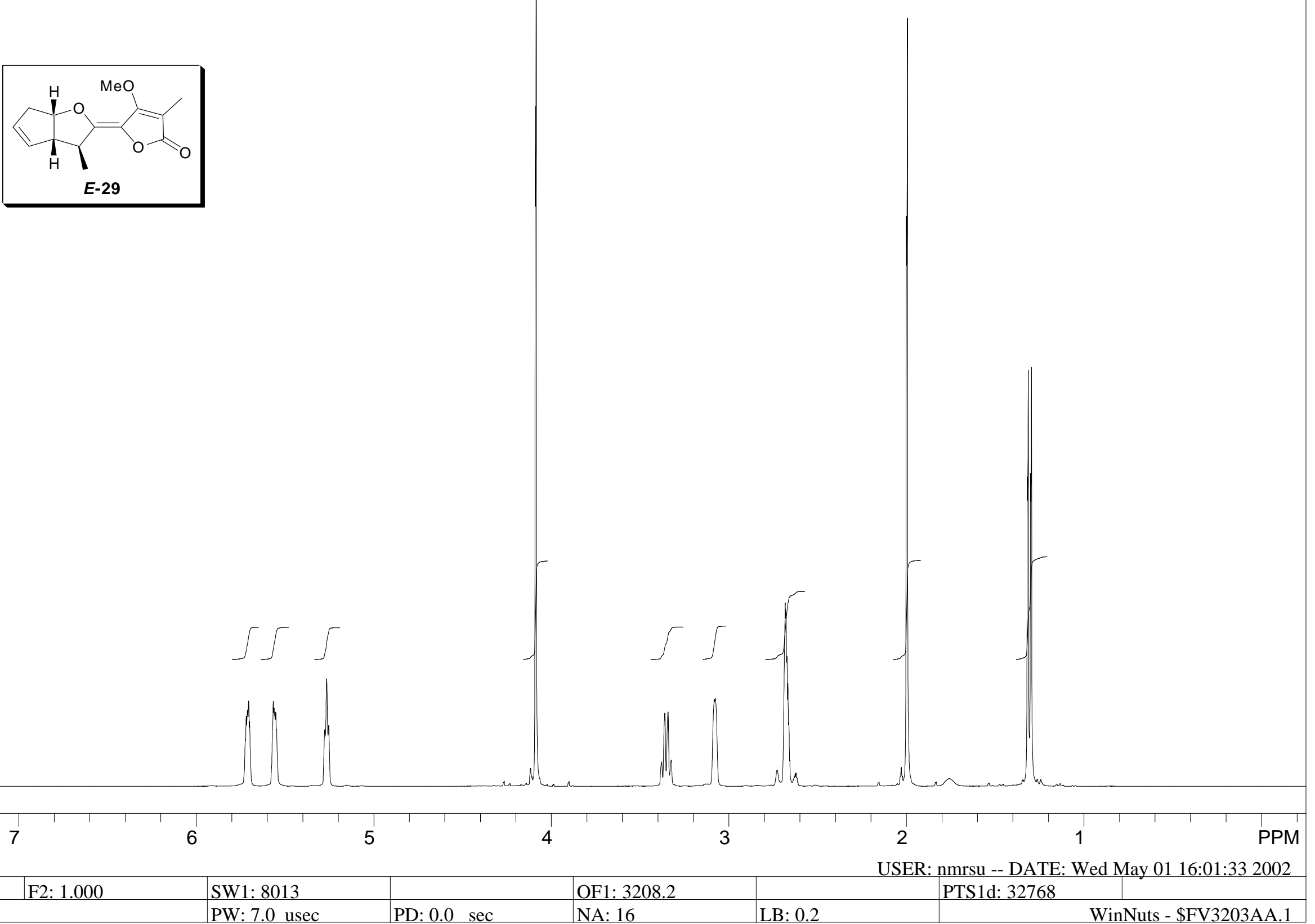


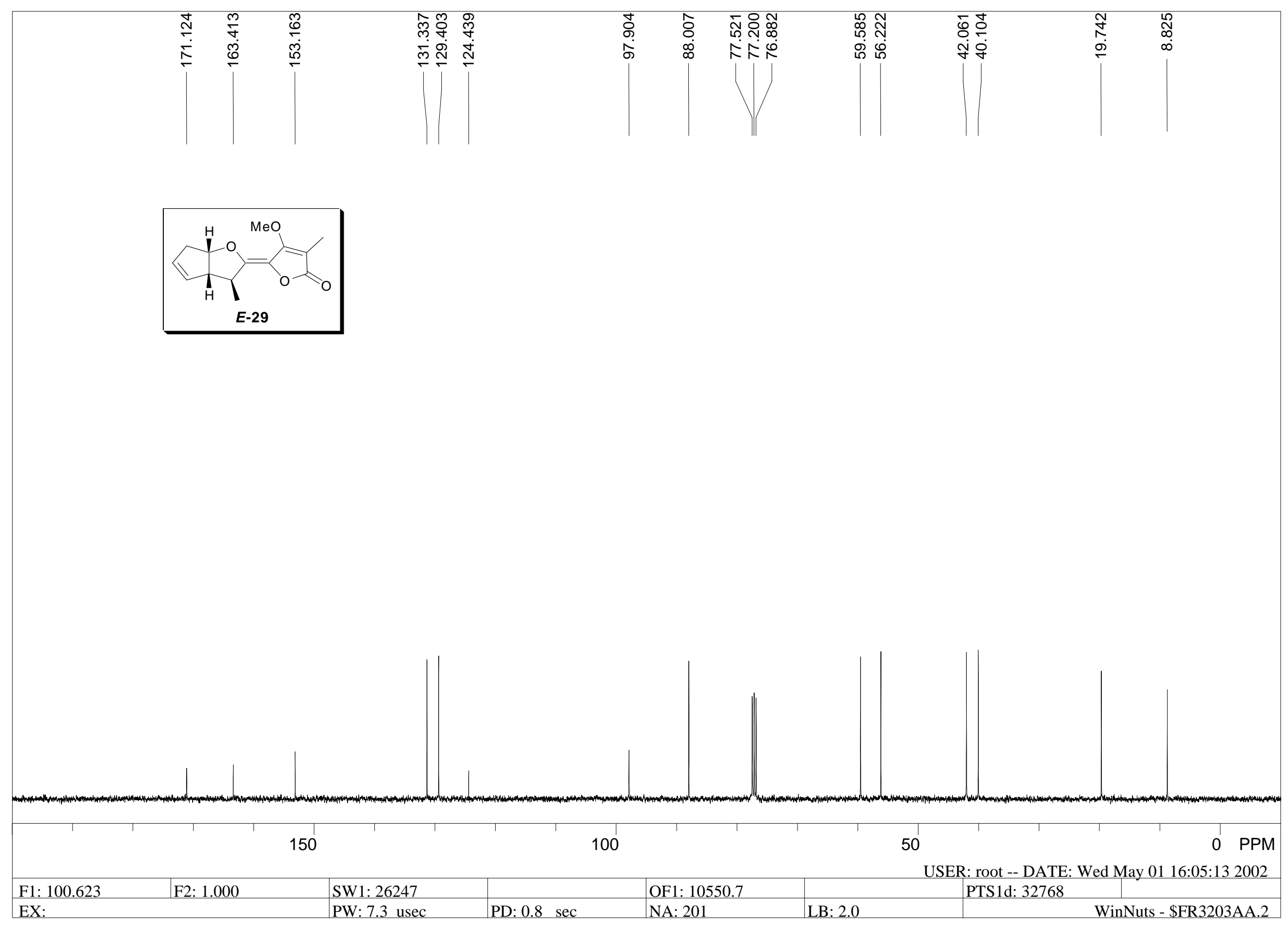




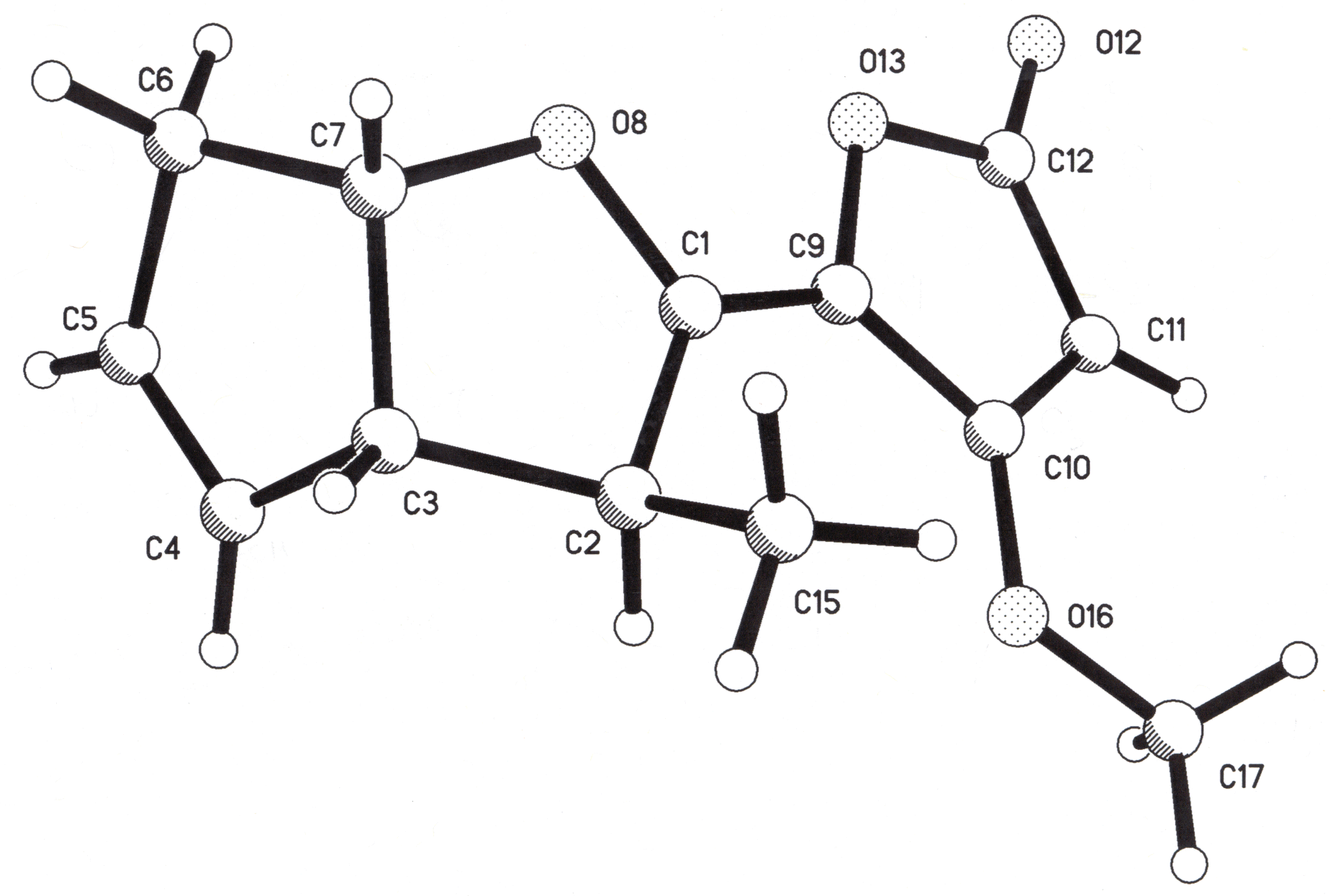

